\title{
Mild Reductive Opening of Aryl Pyranosides Promoted by Scandium (III) Triflate
}

\author{
Hua-Li Qin, Jason T. Lowe and James S. Panek* \\ Department of Chemistry \\ Center for Chemical Methodology and Library Development \\ 590 Commonwealth Avenue, Boston University, \\ Boston, Massachusetts 02215
}

\section{General Information.}

All reactions were carried out in oven or flame-dried glassware under argon atmosphere. Scandium (III) triflate was obtained from Strem. Triethylsilane was obtained from Gelest. All other reagents were purchased from Aldrich. All solvents used for chemical transformations were obtained from a dry solvent system (alumina) and used without further drying. Unless otherwise noted, reactions were magnetically stirred and monitored by thin layer chromatography with Sorbent Technologies $0.20 \mathrm{~mm}$ silica gel $60 \AA$ plates. Flash chromatography was performed on Sorbent Technologies 32-63 $\mu \mathrm{m} 60$ Å silica gel. Yields refer to chromatographically and spectroscopically pure compounds, unless otherwise noted. ${ }^{1} \mathrm{H}$ and ${ }^{13} \mathrm{C}$ NMR spectra were taken in $\mathrm{CDCl}_{3}$ (one in $\mathrm{CD}_{3} \mathrm{OD}$ ) at 400 $\mathrm{MHz}$ and $75 \mathrm{MHz}$, respectively. Chemical shifts are reported in parts per million using the solvent internal standard (chloroform, 7.24 and 77.0 ppm; $\mathrm{CD}_{3} \mathrm{OD}, 3.34$ and 49.86 ppm, respectively). Data are reported as follows: chemical shift, multiplicity (s = singlet, $\mathrm{d}=$ doublet, $\mathrm{t}=$ triplet, $\mathrm{q}=$ quartet, $\mathrm{m}=$ multiplet, $\mathrm{br}=$ broad), coupling constant, integration. Diastereomeric ratios were determined by ${ }^{1} \mathrm{H}$ NMR (400 MHz) analysis of crude mixtures, operating at signal/noise ratio of 200:1. Infrared resonance spectra were 
Mild Reductive Opening of Aryl Pyranosides Promoted by Scandium (III) Triflate

recorded on a Nexus ${ }^{\mathrm{TM}} 670$ FTIR spectrometer. Optical rotations were recorded on an AUTOPOL III digital polarimeter at $589 \mathrm{~nm}$ and reported as follows: concentration $(c$ in g/100mL) and solvent. High resolution mass-spectra were obtained on a Finnagan MAT90 spectrometer at Boston University Mass Spectrometry Laboratory.

\section{General procedure for tetrahydropyran formation from crotylsilanes and aldehyde} via $[4+2]$-annulation: ${ }^{1}$

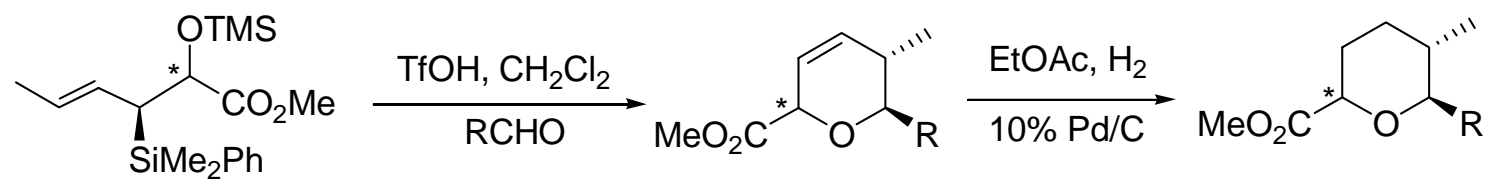

The crotylsilane (either anti-(3S,E)-crotylsilane or syn- $(3 S, E)$-crotylsilane $)^{2}$ (1.1 equiv.) was placed in a round bottom flask equipped with a stir bar and a $0.05 \mathrm{M}$ solution of the appropriate aldehyde solution in $\mathrm{CH}_{2} \mathrm{Cl}_{2}$. The solution was then cooled to $-78{ }^{\circ} \mathrm{C}$ and triflic acid (1 equiv.) was added in one portion. The reaction was allowed to stir for 24 hours at $-78{ }^{\circ} \mathrm{C}$ before a saturated solution of $\mathrm{NaHCO}_{3}$ (equal volume) was added. The solution was allowed to warm to room temperature on its own and extracted three times with $\mathrm{CH}_{2} \mathrm{Cl}_{2}$. The combined organic extracts were dried over $\mathrm{MgSO}_{4}$, filtered and concentrated under vacuum to get a crude oil.

The resulting crude dihydropyrans were placed to round bottom flask under an atmosphere of hydrogen with $10 \% \mathrm{Pd} / \mathrm{C}(0.1$ equiv.) and $10 \mathrm{~mL}$ ethyl acetate and stirred for additional 20 hours. After filtering off $\mathrm{Pd} / \mathrm{C}$ over celite the solutions were concentrated under vacuum to get a crude oil. Product was purified by column chromatography over silica gel with EtOAc/Hexanes(5/95).

(1) For seminal report of [4+2]-annulations involving enantioenriched crotylsilanes see: Huang, H.; Panek, J. S. J. Am. Chem. Soc. 2000, 122, 9836.

(2) (a) For the preparation of anti-(E)-crotylsilane see: Yang, M.; Solomon, J. S.; Panek, J. S. J. Org. Chem. 1993, 58, 1003. (b) For the preparation of syn-(E)-crotylsilane see: Sparks, M. A.; Panek, J. S. J. Org. Chem. 1991, 56, 3431. 


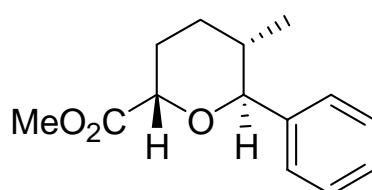

$(2 S, 5 S, 6 S)$-methyl -5-methyl-6-phenyl-tetrahydro-2H-pyran-2-

carboxylate (1a). (Obtained as a colorless oil in 2 step $87 \%$ yield from benzaldehyde and anti-(2R,3S,E)-crotylsilane); $[\alpha]_{\mathrm{D}}^{20}+0.7\left(c\right.$ 1.0, $\left.\mathrm{CHCl}_{3}\right) ;{ }^{1} \mathrm{H} \mathrm{NMR}\left(400 \mathrm{MHz}, \mathrm{CDCl}_{3}\right) \delta$ $7.33(\mathrm{~m}, 5 \mathrm{H}), 4.61(\mathrm{~d}, J=7.8 \mathrm{~Hz}, 1 \mathrm{H}), 4.38(\mathrm{~d}, J=9.2 \mathrm{~Hz}, 1 \mathrm{H}), 3.77(\mathrm{~s}, 3 \mathrm{H}), 2.21(\mathrm{~m}$, 1H), $2.04(\mathrm{~m}, 1 \mathrm{H}), 1.78(\mathrm{~m}, 2 \mathrm{H}), 1.28(\mathrm{~m}, 1 \mathrm{H}), 0.65(\mathrm{~d}, J=6.9 \mathrm{~Hz}, 3 \mathrm{H}) ;{ }^{13} \mathrm{C} \mathrm{NMR}(75$ $\left.\mathrm{MHz} \mathrm{CDCl}_{3}\right) \delta 173.2,141.0,128.5,128.1,128.8,82.7,73.6,52.1,36.2,29.2,27.3$, 18.2 ppm; IR (film) $v_{\max } 3030,2952,2927,1745,1495,1454 \mathrm{~cm}^{-1} ; \mathrm{HRMS}\left(\mathrm{CI}, \mathrm{NH}_{3}\right) \mathrm{m} / z$ calc'd for $\mathrm{C}_{14} \mathrm{H}_{19} \mathrm{O}_{3}[\mathrm{M}+\mathrm{H}]^{+}$235.1334, Found 235.1339.<smiles>COC(C)C1CC[C@@H](C)[C@H](c2ccc3ccccc3c2)O1</smiles>

(2S,5S,6S)-methyl-5-methyl-6-(naphthalen-2-yl)tetrahydro-2H-pyran-2-carboxylate (1b). (Obtained as a colorless oil in 2 step 83\% yield from 2-naphthaldehyde and anti-(2R,3S,E)-crotylsilane); $[\alpha]_{\mathrm{D}}^{20}+7.6$ (c 2.30, $\left.\mathrm{CHCl}_{3}\right) ;{ }^{1} \mathrm{H} \mathrm{NMR}\left(400 \mathrm{MHz}, \mathrm{CDCl}_{3}\right) \delta 7.83(\mathrm{~m}, 3 \mathrm{H}), 7.52(\mathrm{~d}, J=10 \mathrm{~Hz}, 1 \mathrm{H}), 7.45(\mathrm{~m}$, 2H), $7.26(\mathrm{~d}, J=8.0 \mathrm{~Hz}, 1 \mathrm{H}), 4.66(\mathrm{~d}, J=5.6 \mathrm{~Hz}, 1 \mathrm{H}), 4.56(\mathrm{~d}, J=10.0 \mathrm{~Hz}, 1 \mathrm{H}), 3.78(\mathrm{~s}$, 3H), $2.29(\mathrm{~m}, 1 \mathrm{H}), 2.13(\mathrm{~m}, 1 \mathrm{H}), 1.87(\mathrm{~m}, 2 \mathrm{H}), 1.32(\mathrm{~m}, 1 \mathrm{H}), 0.68(\mathrm{~d}, J=6.8 \mathrm{~Hz}, 3 \mathrm{H})$; ${ }^{13} \mathrm{C}$ NMR $\left(75 \mathrm{MHz}, \mathrm{CDCl}_{3}\right) \delta 173.1,138.5,133.5,128.3,128.0,127.8,127.1,127.5$, $126.2,126.0,125.4,82.8,73.7,52.2,36.2,29.2,27.4,18.3 \mathrm{ppm}$; IR (film) $v_{\max } 3052$, 2927, 1744, $1455 \mathrm{~cm}^{-1}$; HRMS(CI, $\left.\mathrm{NH}_{3}\right) \mathrm{m} / z$ calc'd for $\mathrm{C}_{18} \mathrm{H}_{21} \mathrm{O}_{3}[\mathrm{M}+\mathrm{H}]^{+} 285.1491$, Found 285.1489.

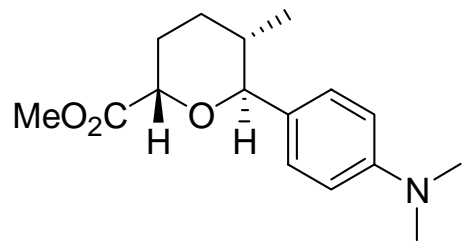

(2S,5S,6S)-methyl 6-(4-(dimethylamino)phenyl)-5-methyltetrahydro-2H-pyran-2-carboxylate (1d). (Obtained as a colorless oil in 2 step $81 \%$ yield from dimethylaminobenzaldehyde and anti-(2R,3S,E)-crotylsilane); $[\alpha]_{\mathrm{D}}^{20}+3.4(c$ 
Mild Reductive Opening of Aryl Pyranosides Promoted by Scandium (III) Triflate

2.70, $\left.\mathrm{CHCl}_{3}\right) ;{ }^{1} \mathrm{H} \mathrm{NMR}\left(400 \mathrm{MHz}, \mathrm{CDCl}_{3}\right) \delta 7.19(\mathrm{~d}, J=8.8 \mathrm{~Hz}, 2 \mathrm{H}), 6.69(\mathrm{~d}, J=8.8$ $\mathrm{Hz}, 2 \mathrm{H}), 4.56$ (d, $J=4.8 \mathrm{~Hz}, 1 \mathrm{H}), 4.25$ (d, $J=8.8 \mathrm{~Hz}, 1 \mathrm{H}), 3.75$ (s, 3H), 2.90 (s, 6H), $2.17(\mathrm{~m}, 1 \mathrm{H}), 1.97(\mathrm{~m}, 1 \mathrm{H}), 1.75(\mathrm{~m}, 2 \mathrm{H}), 1.25$ (dd, $J=12.8,1.8 \mathrm{~Hz}, 1 \mathrm{H}), 0.64(\mathrm{~d}, J=6.4$ $\mathrm{Hz}, 3 \mathrm{H}) ;{ }^{13} \mathrm{C}$ NMR $\left(75 \mathrm{MHz}, \mathrm{CDCl}_{3}\right) \delta 173.3,150.4,128.5,120.1,112.8,82.4,73.7$, 52.1, 41.02, 35.8, 29.4, 27.4, 18.4 ppm; IR (film) $v_{\max }$ 2950, 2927, 1735, 1524, 1445, 1216, 1149, 1037, 909, 810, $728 \mathrm{~cm}^{-1}$; HRMS(CI, NH3) $\mathrm{m} / z$ calc'd for $\mathrm{C}_{16} \mathrm{H}_{23} \mathrm{NNaO}_{3}$ $[\mathrm{M}+\mathrm{Na}]^{+}$300.1576, Found 300.1573.

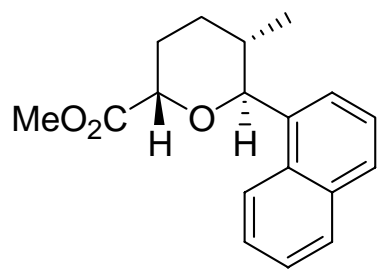

(2S,5S,6S)-methyl 5-methyl-6-(naphthalen-1-yl)-tetrahydro-

2H-pyran-2-carboxylate. (Obtained as a colorless oil in 2 step $85 \%$ yield from 1naphthaldehyde and anti-(2R,3S,E)-crotylsilane); $[\alpha]_{\mathrm{D}}^{20}+6.4\left(\right.$ c 3.10, $\left.\mathrm{CHCl}_{3}\right) ;{ }^{1} \mathrm{H} \mathrm{NMR}$ (400 MHz, $\left.\mathrm{CDCl}_{3}\right) \delta 8.53(\mathrm{~d}, J=4.8 \mathrm{~Hz}, 1 \mathrm{H}), 7.85(\mathrm{~m}, 2 \mathrm{H}), 7.77(\mathrm{~d}, J=9.0 \mathrm{~Hz}, 1 \mathrm{H})$, $7.42(\mathrm{~m}, 2 \mathrm{H}), 7.24-7.37(\mathrm{~m}, 1 \mathrm{H}), 5.30(\mathrm{~m}, 1 \mathrm{H}), 4.68(\mathrm{~d}, J=6.0 \mathrm{~Hz}, 1 \mathrm{H}), 3.79(\mathrm{~s}, 3 \mathrm{H})$, 2.14-2.30 (m, 3H), $1.94(\mathrm{dd}, J=10.0,3.2 \mathrm{~Hz}, 1 \mathrm{H}), 1.40(\mathrm{~m}, 1 \mathrm{H}), 0.68(\mathrm{~d}, J=6.4 \mathrm{~Hz}$, $3 \mathrm{H}) ;{ }^{13} \mathrm{C}$ NMR $\left(75 \mathrm{MHz}, \mathrm{CDCl}_{3}\right) \delta 173.1,138.4,133.5,133.2,128.3,128.2,127.9$, 127.1, 126.2, 126.0, 125.3, 82.8, 73.7, 52.8, 36.2, 29.4, 27.4, 18.3 ppm; IR (film) $v_{\max }$ 3032, 2951, 1741, $1458 \mathrm{~cm}^{-1} ; \operatorname{HRMS}\left(\mathrm{CI}, \mathrm{NH}_{3}\right) \mathrm{m} / z$ calc'd for $\mathrm{C}_{18} \mathrm{H}_{21} \mathrm{O}_{3}[\mathrm{M}+\mathrm{H}]^{+}$ 285.1491, Found 285.1494.

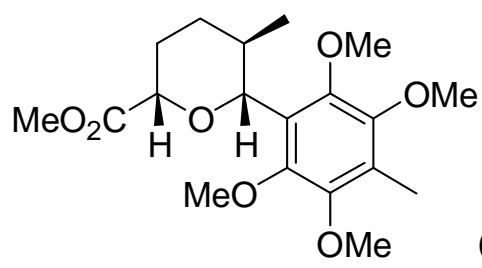

(2S,5R,6R)-5-Methyl-6-(2,3,5,6-tetramethoxy-4-methyl-

phenyl)-tetrahydro-pyran-2-carboxylic acid methyl ester (1i). (Obtained as a colorless oil in 2 step $86 \%$ yield from 2,3,5,6-tetramethoxy-4-methyl-benzaldehyde ${ }^{3}$ and

(3) For the preparation of 2,3,5,6-tetramethoxy-4-methyl-benzaldehyde see: Lowe, J. T.; Panek, J. S. Org. Lett. 2005, 7, 1529 and references therein. 
Mild Reductive Opening of Aryl Pyranosides Promoted by Scandium (III) Triflate

syn-(2R,3S,E)-crotylsilane). $[\alpha]_{\mathrm{D}}^{20}-8.7\left(c \quad 0.70, \mathrm{CHCl}_{3}\right) ;{ }^{1} \mathrm{H} \mathrm{NMR}\left(\mathrm{CDCl}_{3}, 400 \mathrm{MHz}\right): \delta$ $4.46(\mathrm{~d}, J=13.6 \mathrm{~Hz}, 1 \mathrm{H}), 4.11$ (d, $J=15.6 \mathrm{~Hz}, 1 \mathrm{H}), 3.91$ (s, 3H), 3.78 (s, 3H), 3.77 (s, $3 \mathrm{H}), 3.73(\mathrm{~s}, 3 \mathrm{H}), 3.68(\mathrm{~s}, 3 \mathrm{H}), 2.46(\mathrm{~m}, 1 \mathrm{H}), 2.14(\mathrm{~s}, 3 \mathrm{H}), 1.99(\mathrm{~m}, 1 \mathrm{H}), 1.96(\mathrm{~m}, 1 \mathrm{H})$, $1.74(\mathrm{q}, J=16.4 \mathrm{~Hz}, 1 \mathrm{H}), 1.31(\mathrm{q}, J=16.4 \mathrm{~Hz}, 1 \mathrm{H}), 0.65(\mathrm{~d}, J=9.2 \mathrm{~Hz}, 3 \mathrm{H}) ;{ }^{13} \mathrm{C}$ NMR $\left(75 \mathrm{MHz}, \mathrm{CDCl}_{3}\right): \delta 172.1,148.8,148.6,147.6$ 147.2, 125.8, 124.9, 79.4, 77.2, 61.4, 61.0, 60.1, 60.0, 51.8, 32.8, 32.0, 29.6, 18.0, 9.2; IR (film) $v_{\max } 2941,1756,1463 \mathrm{~cm}^{-1}$; HRMS(CI, $\left.\mathrm{NH}_{3}\right) \mathrm{m} / z$ calc'd for $\mathrm{C}_{19} \mathrm{H}_{28} \mathrm{O}_{7}[\mathrm{M}]^{+} 368.1835$, found: 368.1832 .

\section{General procedure for reduction of methyl ester to primary alcohol}

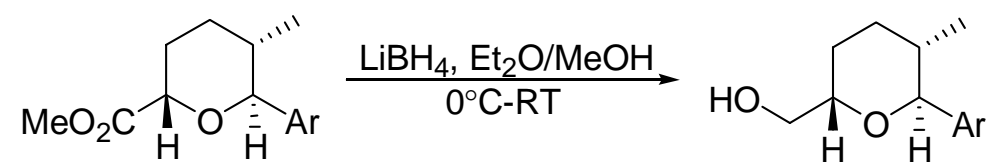

To a solution of ester $(0.1 \mathrm{mmol})$ in $\mathrm{Et}_{2} \mathrm{O}(5.0 \mathrm{~mL})$ at $0{ }^{\circ} \mathrm{C}$ was added $\mathrm{LiBH}_{4}(0.3$ $\mathrm{mmol})$. To the cool solution $\mathrm{MeOH}(0.2 \mathrm{mmol})$ was added dropwise under an atmosphere of argon. The reaction mixture was allowed to warm up to room temperature over 2 hours and stirred at room temperature for an additional 4-6 hours. The mixture was then cooled to $0{ }^{\circ} \mathrm{C}$ and $\mathrm{MeOH}(2.0 \mathrm{~mL})$ was added followed by $\mathrm{H}_{2} \mathrm{O}(2.0 \mathrm{~mL})$ and $1.0 \mathrm{M}$ aqueous solution of $\mathrm{HCl}$. The mixture was stirred at $0{ }^{\circ} \mathrm{C}$ for 20 minutes, extracted three times with $\mathrm{Et}_{2} \mathrm{O}$. The combined organic extracts were dried over $\mathrm{MgSO}_{4}$, filtered, and concentrated under reduced pressure. The residue was purified by flash chromatography over silica gel to yield the desired alcohol.

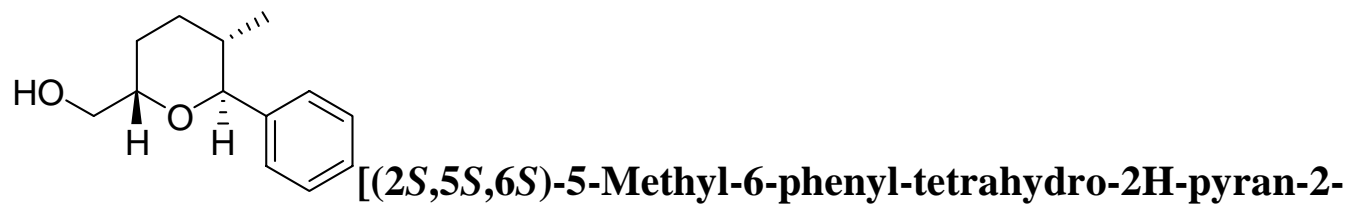

yl]methanol (1f). (Obtained in 99\% yield from ester 1a); $[\alpha]_{\mathrm{D}}^{20}+13.2$ (c 1.62, $\mathrm{CHCl}_{3}$ ); ${ }^{1} \mathrm{H} \mathrm{NMR}\left(400 \mathrm{MHz}, \mathrm{CDCl}_{3}\right) \delta 7.34(\mathrm{~m}, 5 \mathrm{H}), 4.21(\mathrm{~d}, J=8.8 \mathrm{~Hz}, 1 \mathrm{H}), 4.03(\mathrm{~d}, J=7.2 \mathrm{~Hz}$, $1 \mathrm{H}), 3.46(\mathrm{~d}, J=6.8 \mathrm{~Hz}, 2 \mathrm{H}), 1.89(\mathrm{~m}, 2 \mathrm{H}), 1.65(\mathrm{~m}, 1 \mathrm{H}), 1.58(\mathrm{~m}, 1 \mathrm{H}), 1.40(\mathrm{~m}, 1 \mathrm{H})$, $0.75(\mathrm{~d}, J=7.2 \mathrm{~Hz}, 3 \mathrm{H}) ;{ }^{13} \mathrm{C} \mathrm{NMR}\left(75 \mathrm{MHz}, \mathrm{CDCl}_{3}\right) \delta 141.3,128.6,128.1,127.6,79.3$, 
Mild Reductive Opening of Aryl Pyranosides Promoted by Scandium (III) Triflate

73.2, 61.6, 35.4, 27.7, 25.1, 18.4 ppm; IR (film) $v_{\max } 3392,2927,1493,1454 \mathrm{~cm}^{-1}$; HRMS(CI, NH3) $m / z$ calc'd for $\mathrm{C}_{13} \mathrm{H}_{18} \mathrm{NaO}_{2}[\mathrm{M}+\mathrm{Na}]^{+}$229.1204, Found 229.1206.<smiles>C[C@H]1CC[C@@H](CO)O[C@H]1c1cccc2ccccc12</smiles>

[(2S,5S,6S)-5-Methyl-6-(naphthalen-1-yl)-tetrahydro-2H-pyran-

2-yl]methanol (1g). (Obtained in 96\% yield from ester (see SI-4)); $[\alpha]_{\mathrm{D}}^{20}+50.3$ (c 2.90, $\left.\mathrm{CHCl}_{3}\right) ;{ }^{1} \mathrm{H} \mathrm{NMR}\left(400 \mathrm{MHz}, \mathrm{CDCl}_{3}\right) \delta 8.36(\mathrm{~d}, J=8.4 \mathrm{~Hz}, 1 \mathrm{H}), 7.86(\mathrm{~d}, J=8.0 \mathrm{~Hz}$, $1 \mathrm{H}), 7.80(\mathrm{~d}, J=8.0 \mathrm{~Hz}, 1 \mathrm{H}), 7.51(\mathrm{~m}, 3 \mathrm{H}), 7.32(\mathrm{~m}, 1 \mathrm{H}), 4.98(\mathrm{~d}, J=8.8 \mathrm{~Hz}, 1 \mathrm{H}), 4.18$ $(\mathrm{d}, J=4.8 \mathrm{~Hz}, 1 \mathrm{H}), 3.51(\mathrm{~d}, J=6.6 \mathrm{~Hz}, 2 \mathrm{H}), 2.26(\mathrm{~m}, 1 \mathrm{H}), 2.06($ br m, 1H), $1.81(\mathrm{~m}, 1 \mathrm{H})$, $1.68(\mathrm{~m}, 1 \mathrm{H}), 1.54(\mathrm{~m}, 1 \mathrm{H}), 1.31(\mathrm{~m}, 1 \mathrm{H}), 0.77(\mathrm{~d}, J=6.4 \mathrm{~Hz}, 3 \mathrm{H}) ;{ }^{13} \mathrm{C}$ NMR $(75 \mathrm{MHz}$, $\left.\mathrm{CDCl}_{3}\right) \delta 136.7,134.3,132.1,129.1,128.7,128.6,126.2,125.7,125.4,124.8,76.5,73.6$, 61.4, 35.1, 28.4, 25.4, 18.9 ppm; IR (film) v $v_{\max } 3401,3048,2926,1638,1597,1456 \mathrm{c} \mathrm{m}^{-1}$; HRMS(CI, NH3) $m / z$ calc'd for $\mathrm{C}_{17} \mathrm{H}_{20} \mathrm{NaO}_{2}[\mathrm{M}+\mathrm{Na}]^{+} 279.1362$, Found 279.1366.<smiles>COc1c(C)c(OC)c(OC)c([C@]2(C)O[C@H](CO)[C@@H](C)[C@H]([OH+])[C@H]2C)c1OC</smiles>

[(2S,3S,4R,5S,6R)-4-(tert-Butyl-dimethyl-silanyloxy)-3,5-

dimethyl-6-(2,3,5,6-tetramethoxy-4-methyl-phenyl)-tetrahydro-pyran-2-yl]-

methanol (1j). (Obtained in $95 \%$ yield from known ester. $\left.{ }^{3}\right)[\alpha]_{\mathrm{D}}^{20}+30.1\left(c 0.83, \mathrm{CHCl}_{3}\right)$; ${ }^{1} \mathrm{H}$ NMR $\left(\mathrm{CDCl}_{3}, 400 \mathrm{MHz}\right): \delta 4.45$ (d, $\left.J=10.8 \mathrm{~Hz}, 1 \mathrm{H}\right), 3.84(\mathrm{~s}, 3 \mathrm{H}), 3.74(\mathrm{~s}, 3 \mathrm{H}), 3.72$ (s, 3H), 3.76 (s, 3H), 3.73 (obsc. m, 1H), 3.65 (dt, $J=8.8,2.8 \mathrm{~Hz}, 1 \mathrm{H}), 3.47$ (td, $J=10.4$, $2.8 \mathrm{~Hz}, 2 \mathrm{H}), 2.54$ (m, 1H), 2.15 (s, 3H), 1.84 (m, 1H), 1.80 (br. s, 1H), 1.02 (d, J = 6.8 $\mathrm{Hz}, 3 \mathrm{H}), 0.89(\mathrm{~s}, 9 \mathrm{H}), 0.64(\mathrm{~d}, J=6.4 \mathrm{~Hz}, 3 \mathrm{H}), 0.06(\mathrm{~s}, 6 \mathrm{H}) ;{ }^{13} \mathrm{C}$ NMR $\left(75 \mathrm{MHz}, \mathrm{CDCl}_{3}\right)$ : $\delta 148.7,148.5,147.6,147.2,125.9,124.9,80.4,78.1,77.7,64.4,61.1,61.0,60.2,60.1$, 37.7, 35.2, 25.8, 18.1, 14.3, 9.3, 6.5, -4.2, -4.8; IR (film) $v_{\max } 3462,2935,1464 \mathrm{~cm}^{-1}$; $\mathrm{HRMS}\left(\mathrm{CI}, \mathrm{NH}_{3}\right) \mathrm{m} / z$ calc'd for $\mathrm{C}_{25} \mathrm{H}_{45} \mathrm{O}_{7} \mathrm{Si}[\mathrm{M}+\mathrm{H}]^{+}$485.2935, found: 485.2850 . 


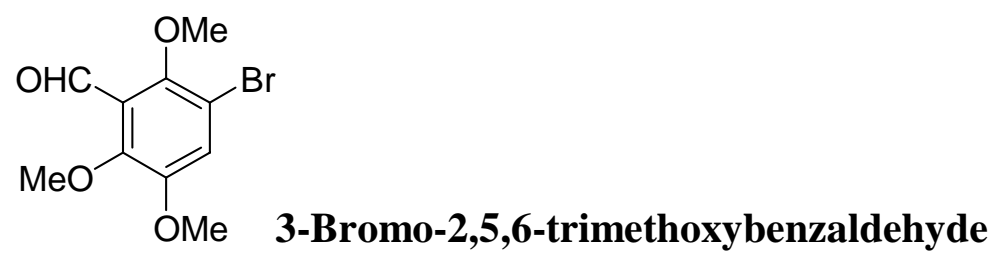

To a solution of 2,3,6-trimethoxybenzaldehyde ${ }^{4}(14.0 \mathrm{~g}, 71.4 \mathrm{mmol})$ in acetic acid (350 $\mathrm{mL})$ was added sequentially sodium acetate $(8.62 \mathrm{~g}, 105 \mathrm{mmol})$ and the dropwise addition of bromine $(4.40 \mathrm{~mL}, 85.4 \mathrm{mmol})$. The reaction was stirred for 30 minutes before acetic acid was concentrated and residue was diluted with water. The aqueous layer was extracted with methylene chloride. The combined organic layers were dried with $\mathrm{MgSO}_{4}$, filtered over silica gel, and concentrated under reduced pressure. The residue was purified by flash chromatography over silica gel with (hexanes/EtOAc: 90/10) to yield $87 \%$ of 4-bromo-2,3,6-trimethoxybenzaldehyde (16.9 g, $62.1 \mathrm{mmol})$ as a light yellow solid. ${ }^{1} \mathrm{H}$ NMR (400 MHz, $\left.\mathrm{CDCl}_{3}\right) \delta 10.32(\mathrm{~s}, 1 \mathrm{H}), 7.26$ (s, 1H), 3.89 (s, 3H), $3.84(\mathrm{~s}, 3 \mathrm{H}), 3.82(\mathrm{~s}, 3 \mathrm{H}) ;{ }^{13} \mathrm{C} \mathrm{NMR}\left(75 \mathrm{MHz} \mathrm{CDCl}_{3}\right) \delta 189.1,151.4,151.0,150.2$, 123.8, 121.6, 112.4, 62.8, 62.3, 56.7 ppm; IR (film) $v_{\max } 2939,2843,1695,1563 \mathrm{~cm}^{-1}$; $\mathrm{mp}=63-65^{\circ} \mathrm{C}$. HRMS(CI, $\left.\mathrm{NH} 3\right) \mathrm{m} / z$ calc'd for $\mathrm{C}_{10} \mathrm{H}_{12} \mathrm{BrO}_{4}[\mathrm{M}+\mathrm{H}]^{+}$274.9919, Found 274.9910 .<smiles>COc1cc(Br)c(OC)c([C@H]2O[C@H](C(C)=O)C=C[C@@H]2C)c1OC</smiles>

(2S,5S,6S)-Methyl-6-(3-bromo-2,5,6-trimethoxyphenyl)-

\section{5-methyl-5,6-dihydro-2H-pyran-2-carboxylate}

The $(2 R, 3 S)-(E)$-methyl-3-(dimethyl(phenyl)silyl)-2-(trimethylsilyloxy)-hex-4-enoate (385.7 $\mathrm{mg}, 1.10 \mathrm{mmol}$ ) was placed in a round bottom flask equipped with a stir bar, 20 $\mathrm{ml} \mathrm{CH}_{2} \mathrm{Cl}_{2}$, and 3-bromo-2,5,6-trimethoxybenzaldehyde (275.5 mg, $\left.1.01 \mathrm{mmol}\right)$. The mixture was then cooled to $-78{ }^{\circ} \mathrm{C}$ and triflic acid $(151.3 \mathrm{mg}, 1.0 \mathrm{mmol})$ was added in one portion. The reaction was allowed to stir for 24 hours at $-78{ }^{\circ} \mathrm{C}$ before a saturated

(4) For preparation of 2,3,6-trimethoxybenzaldehyde and 2,3,6-trimethoxy-4nitrobenzaldehyde, see Andrus M. B.; Meredith E. L.; Hicken E. J.; Simmons B. L.; Glancey R. R.; Ma W. J. Org. Chem. 2003, 68, 8162. 
Mild Reductive Opening of Aryl Pyranosides Promoted by Scandium (III) Triflate

solution of $\mathrm{NaHCO}_{3}$ (equal volume) was added. The solution was allowed to warm to room temperature on its own. The aqueous layer was then extracted with additional $\mathrm{CH}_{2} \mathrm{Cl}_{2}(3 \mathrm{x})$. The combined organic extracts were placed over $\mathrm{MgSO}_{4}$, filtered and concentrated under vacuum to get a crude oil. The resulting crude dihydropyran was purified by column chromatography over silica gel with (hexanes/ $\mathrm{CH}_{2} \mathrm{Cl}_{2} / \mathrm{EtOH}$ : 75/24/1) to yield $78 \%$ of pure dihydropyran $(312.8 \mathrm{mg}, 0.78 \mathrm{mmol})$ as light yellow oil. $[\alpha]_{\mathrm{D}}^{20}-48.2$ (c 3.60, $\left.\mathrm{CHCl}_{3}\right) ;{ }^{1} \mathrm{H}$ NMR (400 MHz, $\left.\mathrm{CDCl}_{3}\right) \delta 7.03(\mathrm{~s}, 1 \mathrm{H}), 5.88(\mathrm{~m}, 2 \mathrm{H}), 4.91$ (d, $J=$ $12 \mathrm{~Hz}, 1 \mathrm{H}), 4.73$ (s, 1H), 3.87 (s, 3H), 3.84 (s, 3H), 3.82 (s, 3H), 3.74 (s, 3H), 3.12 (m, $1 \mathrm{H}), 0.73(\mathrm{~d}, J=9.8 \mathrm{~Hz}, 3 \mathrm{H}) ;{ }^{13} \mathrm{C} \mathrm{NMR}\left(75 \mathrm{MHz}, \mathrm{CDCl}_{3}\right) \delta 172.1,150.7,149.3,132.2$, 129.6, 122.5, 119.2, 76.8, 75.0, 74.3, 62.7, 61.3, 57.9, 56.8, 54.2, 31.8, 18.1 ppm; IR (film) $v_{\max } 2956,1742,1563,1475,1425 \mathrm{~cm}^{-1}$; HRMS(CI, NH3) $\mathrm{m} / z$ calc'd for $\mathrm{C}_{17} \mathrm{H}_{22} \mathrm{BrO}_{6}[\mathrm{M}+\mathrm{H}]^{+}$401.0600, Found 401.0604.

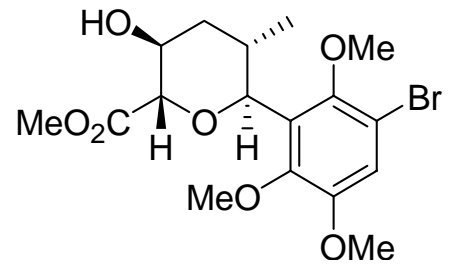

(2S,3S,5S,6S)-Methyl-6-(3-bromo-2,5,6-trimethoxyphenyl)3-hydroxy-5-methyl-tetrahydro-2H-pyran-2-carboxylate

(2S,5S,6S)-Methyl-6-(3-bromo-2,5,6-trimethoxyphenyl)-5-methyl-5,6-dihydro-2H-pyran2-carboxylate $(200.3 \mathrm{mg}, 0.5 \mathrm{mmol})$ was placed in a round bottom flask quipped with $10 \mathrm{ml}$ THF. The solution was cooled to $0^{\circ} \mathrm{C}$ before borane-dimethyl sulfide complex (2.0M in THF, $0.8 \mathrm{~mL}$ ) was added dropwise under an atmosphere of argon. The reaction mixture was allowed to warm up to room temperature and stirred for 6 hours. The mixture was cooled to $0{ }^{\circ} \mathrm{C}$ then a solution of sodium hydroxide in water $(1.0 \mathrm{M}, 5.0 \mathrm{~mL})$ and hydrogen peroxide ( $30 \%$ aqueous solution, $0.8 \mathrm{~mL}$ ) were added. The mixture was stirred at $0{ }^{\circ} \mathrm{C}$ for 30 minutes and at room temperature for 1 hours before $\mathrm{H}_{2} \mathrm{O}$ was added to dilute. The mixture was extracted five times with $\mathrm{Et}_{2} \mathrm{O}$ (aqueous phase was saturated with solid $\mathrm{NaCl}$ each time). The combined organic extracts were dried over $\mathrm{MgSO}_{4}$, filtered, and concentrated under reduced pressure to get crude alcohol. The alcohol was purified by flash chromatography over silica gel with (hexanes/EtOAc/Et ${ }_{2} \mathrm{O}: 80 / 5 / 15$ ) to 
Mild Reductive Opening of Aryl Pyranosides Promoted by Scandium (III) Triflate

get colorless oil $(165.3 \mathrm{mg}, 79 \%)$. $[\alpha]_{\mathrm{D}}^{20}+3.2\left(c 0.60, \mathrm{CHCl}_{3}\right) ;{ }^{1} \mathrm{H}$ NMR $(400 \mathrm{MHz}$, $\left.\mathrm{CDCl}_{3}\right) \delta 7.04(\mathrm{~s}, 1 \mathrm{H}), 5.02(\mathrm{~d}, J=14.8 \mathrm{~Hz}, 1 \mathrm{H}), 4.53(\mathrm{~s}, 1 \mathrm{H}), 4.30(\mathrm{~s}, 1 \mathrm{H}), 3.90(\mathrm{~s}, 3 \mathrm{H})$, 3.86 (s, 3H), 3.80 (s, 3H), 3.78 (s, 3H), 3.01 (m, 1H), $2.58(\mathrm{~m}, 1 \mathrm{H}), 1.94(\mathrm{~m}, 1 \mathrm{H}), 1.40$ $(\mathrm{m}, 1 \mathrm{H}), 0.64(\mathrm{~d}, J=8.8 \mathrm{~Hz}, 3 \mathrm{H}) ;{ }^{13} \mathrm{C}$ NMR $\left(75 \mathrm{MHz}, \mathrm{CDCl}_{3}\right) \delta 171.8,150.9,149.1$, 129.6, 116.2, 112.6, 75.7, 75.0, 74.1, 73.7, 62.7, 61.3, 56.3, 52.2, 34.3, 26.7, 18.9 ppm; IR (film) $v_{\max } 3450,2945,1742,1475 \mathrm{~cm}^{-1}$; HRMS(CI, NH3) $\mathrm{m} / z$ calc'd for $\mathrm{C}_{17} \mathrm{H}_{24} \mathrm{BrO}_{7}$ $[\mathrm{M}+\mathrm{H}]^{+}$419.0705, Found 419.0710.<smiles>COC(=O)[C@H]1O[C@H](c2c(OC)c(Br)cc(OC)c2OC)[C@H](C)C[C@@H]1OC(=O)[C@H](OC(C)=O)c1ccccc1</smiles>

(2S,3S,5S,6S)-Methyl-3-[(R)-2-acetoxy-2-phenylacetoxy]6-(3-bromo-2,5,6-trimethoxyphenyl)-5-methyl-tetrahydro-2H-pyran-2-carboxylate

(2S,3S,5S,6S)-Methyl-6-(3-bromo-2,5,6-trimethoxyphenyl)-3-hydroxy-5-methyltetrahydro-2H-pyran-2-carboxylate $(41.1 \mathrm{mg}, 0.098 \mathrm{mmol}$ ) was added into a round bottom flask along with DCC (35.5 mg, $0.172 \mathrm{mmol})$, DMAP (3.0 mg, $0.026 \mathrm{mmol})$ and $\mathrm{CH}_{2} \mathrm{Cl}_{2}(3 \mathrm{~mL})$. A solution of $(R)$-acetoxymandelic acid (19.7 mg, $\left.0.10 \mathrm{mmol}\right)$ in $\mathrm{CH}_{2} \mathrm{Cl}_{2}$ $(1.0 \mathrm{~mL})$ was then added at room temperature and allowed to stir overnight. The solution was filtered and concentrated under vacuum to get cloudy oil. The oil was then purified over silica gel (hexanes/THF/EtOAc: $90 / 5 / 5)$ to get a clear oil $(46.6 \mathrm{mg}, 80 \%)$. $[\alpha]_{\mathrm{D}}^{20}+$ $17.6\left(\right.$ c $\left.2.50, \mathrm{CHCl}_{3}\right) ;{ }^{1} \mathrm{H} \mathrm{NMR}\left(400 \mathrm{MHz}, \mathrm{CDCl}_{3}\right) \delta 7.48(\mathrm{~m}, 2 \mathrm{H}), 7.38(\mathrm{~m}, 3 \mathrm{H}), 7.02(\mathrm{~s}$, 1H), $5.93(\mathrm{~s}, 1 \mathrm{H}), 5.46(\mathrm{~s}, 1 \mathrm{H}), 4.87(\mathrm{~d}, J=10.8 \mathrm{~Hz}, 1 \mathrm{H}), 4.64(\mathrm{~s}, 1 \mathrm{H}), 3.86(\mathrm{~s}, 3 \mathrm{H}), 3.81$ (s, 3H), $3.76(\mathrm{~s}, 3 \mathrm{H}), 3.71(\mathrm{~s}, 3 \mathrm{H}), 2.68(\mathrm{~m}, 1 \mathrm{H}), 2.21(\mathrm{~s}, 3 \mathrm{H}), 1.77$ (dd, $J=13.6,2.3 \mathrm{~Hz}$, $1 \mathrm{H}), 1.36(\mathrm{~m}, 1 \mathrm{H}), 0.47(\mathrm{~d}, J=6.4 \mathrm{~Hz}, 3 \mathrm{H}) ;{ }^{13} \mathrm{C} \mathrm{NMR}\left(75 \mathrm{MHz}, \mathrm{CDCl}_{3}\right) \delta 170.5,169.8$, 168.4, 150.0, 148.2, 133.3, 129.3, 128.9, 128.8, 127.9, 127.4, 116.1, 75.7, 75.4, 74.8, 70.1, 69.8, 62.1, 61.4, 56.1, 52.3, 33.5, 26.4, 20.6, 17.2 ppm; IR (film) $v_{\max } 2941,1749$, 
1476, $1426 \mathrm{~cm}^{-1}$; HRMS(CI, NH3) $m / z$ calc'd for $\mathrm{C}_{27} \mathrm{H}_{32} \mathrm{BrO}_{10}[\mathrm{M}+\mathrm{H}]^{+}$595.1179, Found 595.1174 .<smiles>COc1cc(Br)c(OC)c([C@@H]2C[C@@H](OC(=O)[C@H](OC(C)=O)c3ccccc3)C[C@@H](C(C)=O)O2)c1OC</smiles>

(2S,3S,5S,6S)-Methyl-3-[(s)-2-acetoxy-2-

phenylacetoxy]-6-(3-bromo-2,5,6-trimethoxyphenyl)-5-methyl-tetrahydro-2H-

pyran-2-carboxylate

(2S,3S,5S,6S)-Methyl-6-(3-bromo-2,5,6-trimethoxyphenyl)-3-hydroxy-5-methyltetrahydro-2H-pyran-2-carboxylate $(41.1 \mathrm{mg}, 0.098 \mathrm{mmol})$ was added into a round bottom flask along with DCC (35.5 mg, $0.172 \mathrm{mmol})$, DMAP (3.0 mg, $0.026 \mathrm{mmol})$ and $\mathrm{CH}_{2} \mathrm{Cl}_{2}(3 \mathrm{~mL})$. A solution of $(S)$-acetoxymandelic acid $(19.7 \mathrm{mg}, 0.10 \mathrm{mmol})$ in $\mathrm{CH}_{2} \mathrm{Cl}_{2}$ $(1.0 \mathrm{~mL})$ was then added at room temperature. The reaction was allowed to stir overnight at room temperature. The solution was filtered and concentrated under vacuum to get cloudy oil. The oil was then purified over silica gel (hexanes/THF/EtOAc: $90 / 5 / 5)$ to get a clear oil $(46.6 \mathrm{mg}, 80 \%) \cdot[\alpha]_{\mathrm{D}}^{20}+31.5\left(\right.$ c 2.85, $\left.\mathrm{CHCl}_{3}\right) ;{ }^{1} \mathrm{H} \mathrm{NMR}\left(400 \mathrm{MHz}, \mathrm{CDCl}_{3}\right) \delta$ 7.50 (m, 2H), 7.38 (m, 3H), 7.02 (s, 1H), 5.94 (s, 1H), 5.49 (s, 1H), 4.85 (d, J= 10.8 Hz, 1H), 4.41 (s, 1H), 3.80 (s, 3H), 3.78 (s, 3H), 3.0 (br. s, 6H), 2.88 (m, 1H), 2.16 (s, 3H), $2.10(\mathrm{dd}, J=13.8,2.0 \mathrm{~Hz}, 1 \mathrm{H}), 1.51(\mathrm{~m}, 1 \mathrm{H}), 0.57(\mathrm{~d}, J=6.4 \mathrm{~Hz}, 3 \mathrm{H}) ;{ }^{13} \mathrm{C} \mathrm{NMR}(75$ $\left.\mathrm{MHz} \mathrm{CDCl}_{3}\right) \delta 170.3,169.8,168.4,149.9,147.4,133.4,129.5,128.9,128.5,127.9$, $126.7,116.2,111.8,76.5,75.7,74.8,70.1,61.9,61.2,56.1,52.3,33.7,26.4,20.7,17.3$ ppm; IR (film) $v_{\max }$ 2944, 1747, 1474, $1373 \mathrm{~cm}^{-1}$; HRMS(CI, NH3) $\mathrm{m} / z$ calc'd for $\mathrm{C}_{27} \mathrm{H}_{32} \mathrm{BrO}_{10}[\mathrm{M}+\mathrm{H}]^{+}$595.1179, Found 595.1181. 
<smiles>COc1cc(Br)c(OC)c([C@H]2OC(C(C)=O)[C@@H](C)C[C@H]2C)c1OC</smiles>

[(2S,3S,5S,6S)-Methyl-6-(3-bromo-2,5,6-

trimethoxyphenyl)]-3-methoxy-5-methyl-tetrahydro-2H-pyran-2-carboxylate (1e).

A flame-dried $50 \mathrm{~mL}$ round bottom flask was charged with (2S,3S,5S,6S)-Methyl-6-(3bromo-2,5,6-trimethoxyphenyl)-3-hydroxy-5-methyl-tetrahydro-2H-pyran-2-carboxylate (125.2 mg, $0.30 \mathrm{mmol}$ ), $20 \mathrm{~mL}$ of dry $\mathrm{CH}_{2} \mathrm{Cl}_{2}$, proton sponge (320.1 $\mathrm{mg}, 1.5 \mathrm{mmol}$ ) and $4 \AA$ molecular sieves $(300 \mathrm{mg})$ were added followed by $\mathrm{Me}_{3} \mathrm{O}^{+} \mathrm{BF}_{4}^{-}(222.4 \mathrm{mg}, 1.5$ mmol). The resulting heterogeneous, light yellow solution was stirred for 12 hours at room temperature. The mixture was then filtered over celite and purified by column chromatography over silica gel with (hexanes/ $\mathrm{CH}_{2} \mathrm{Cl}_{2} / \mathrm{Et}_{2} \mathrm{O}: 70 / 25 / 5$ ) to get $1 \mathrm{e}$ as colorless oil $(125.7 \mathrm{mg}, 97 \%) .[\alpha]_{\mathrm{D}}^{20}+12.6$ (c 5.60, $\left.\mathrm{CHCl}_{3}\right) ;{ }^{1} \mathrm{H} \mathrm{NMR}\left(400 \mathrm{MHz}, \mathrm{CDCl}_{3}\right)$ $\delta 7.02(\mathrm{~s}, 1 \mathrm{H}), 4.92(\mathrm{~d}, J=6.8 \mathrm{~Hz}, 1 \mathrm{H}), 4.63(\mathrm{~s}, 1 \mathrm{H}), 3.86(\mathrm{~s}, 3 \mathrm{H}), 3.81(\mathrm{~s}, 3 \mathrm{H}), 3.79(\mathrm{~s}$, $3 \mathrm{H}), 3.78(\mathrm{~s}, 3 \mathrm{H}), 3.70(\mathrm{~m}, 1 \mathrm{H}) 3.41(\mathrm{~s}, 3 \mathrm{H}), 2.91(\mathrm{~m}, 1 \mathrm{H}), 2.05(\mathrm{~m}, 1 \mathrm{H}), 1.24(\mathrm{~m}, 1 \mathrm{H})$, $0.57(\mathrm{~d}, J=7.2 \mathrm{~Hz}, 3 \mathrm{H}) ;{ }^{13} \mathrm{C} \mathrm{NMR}\left(75 \mathrm{MHz}, \mathrm{CDCl}_{3}\right) \delta 171.8,150.3,148.8,129.6,116.3$, 112.7, 75.7, 75.0, 74.1, 73.7, 62.3, 61.5, 56.4, 56.3, 52.2, 33.7, 26.0, 18.0 ppm; IR (film) $v_{\max } 2938,1746,1475,1425 \mathrm{~cm}^{-1}$; $\operatorname{HRMS}(\mathrm{CI}, \mathrm{NH} 3) \mathrm{m} / z$ calc'd for $\mathrm{C}_{18} \mathrm{H}_{26} \mathrm{BrO}_{7}[\mathrm{M}+\mathrm{H}]^{+}$ 433.0862, Found 433.0864.<smiles>COc1cc(Br)c(OC)c([C@H]2O[C@H](CO)[C@@H](OC)C[C@H]2C)c1OC</smiles>

[(2R,3S,5S,6S)-6-(3-Bromo-2,5,6-trimethoxyphenyl)-3-

methoxy-5-methyl-tetrahydro-2H-pyran-2-yl]methanol (1h). (See the general procedure for reduction of methyl ester to alcohol on SI-5 to obtain $98 \%$ yield from 1e). $[\alpha]_{\mathrm{D}}^{20}+22.8\left(c 3.12, \mathrm{CHCl}_{3}\right) ;{ }^{1} \mathrm{H} \mathrm{NMR}\left(400 \mathrm{MHz}, \mathrm{CDCl}_{3}\right) \delta 7.03(\mathrm{~s}, 1 \mathrm{H}), 4.67(\mathrm{~d}, J=$ $14.4 \mathrm{~Hz}, 1 \mathrm{H}), 4.13(\mathrm{~m}, 2 \mathrm{H}), 3.85$ (s, 3H), 3.82 (s, 3H), 3.79 (s, 3H), $3.70(\mathrm{~m}, 2 \mathrm{H}), 3.38$ (s, 3H), $2.91(\mathrm{~m}, 1 \mathrm{H}), 2.22($ br s, 1H), $2.02(\mathrm{~m}, 1 \mathrm{H}), 1.39(\mathrm{~m}, 1 \mathrm{H}), 0.65(\mathrm{~d}, J=9.2 \mathrm{~Hz}$, 
Mild Reductive Opening of Aryl Pyranosides Promoted by Scandium (III) Triflate

$3 \mathrm{H}) ;{ }^{13} \mathrm{C}$ NMR $\left(75 \mathrm{MHz}, \mathrm{CDCl}_{3}\right) \delta 150.3,148.3,129.6,116.7,116.3,112.1,75.7,74.5$, 72.6, 62.3, 61.6, 59.9, 56.4, 52.2, 33.9, 26.4, 18.2 ppm; IR (film) $v_{\max } 3430,2938,1746$, 1475, $1425 \mathrm{~cm}^{-1}$; HRMS(CI, NH3) $\mathrm{m} / z$ calc'd for $\mathrm{C}_{17} \mathrm{H}_{25} \mathrm{BrNaO}_{6}[\mathrm{M}+\mathrm{Na}]^{+} 427.0732$, Found 427.0735.<smiles>COc1c(Br)cc(Br)cc1[C@@H]1O[C@H](Cc2ccccc2)C=C[C@H]1C</smiles>

(2S,5S,6S)-6-(3-Benzyloxy-5-bromo-2-methoxy-phenyl)-5methyl-5,6-dihydro-2H-pyran-2-carboxylic acid methyl ester (8b).

(2R,3S)-(E)-Methyl-3-(dimethyl(phenyl)silyl)-2-(trimethylsilyloxy)-hex-4-enoate $(385.7 \mathrm{mg}, 1.10 \mathrm{mmol}$ ) was placed in a round bottom flask equipped with a stir bar, 20 $\mathrm{mL} \mathrm{CH}_{2} \mathrm{Cl}_{2}$, and 3-(benzyloxy)-5-bromo-2-methoxybenzaldehyde ${ }^{5}$ (320.5 mg, 1.01 mmol). The mixture was then cooled to $-78{ }^{\circ} \mathrm{C}$ and triflic acid $(151.3 \mathrm{mg}, 1.00 \mathrm{mmol})$ was added in one portion. The reaction was allowed to stir for 24 hours at $-78{ }^{\circ} \mathrm{C}$ before a saturated solution of $\mathrm{NaHCO}_{3}$ (equal volume) was added. The solution was allowed to warm to room temperature on its own. The aqueous layer was then extracted with additional $\mathrm{CH}_{2} \mathrm{Cl}_{2}(3 \mathrm{x})$. The combined organic extracts were dried over $\mathrm{MgSO}_{4}$, filtered and concentrated under vacuum to get a crude oil. The resulting crude dihydropyran was purified by column chromatography over silica gel with (hexanes/EtOAc: $95 / 5$ ) to get $87 \%$ of pure product $(388.3 \mathrm{mg}, 0.87 \mathrm{mmol})$ as colorless oil. $[\alpha]_{D}^{20}-24.9$ (c 1.02 , $\left.\mathrm{CHCl}_{3}\right) ;{ }^{1} \mathrm{H} \mathrm{NMR}\left(\mathrm{CDCl}_{3}, 400 \mathrm{MHz}\right): \delta 7.43-7.30(\mathrm{~m}, 5 \mathrm{H}), 7.17(\mathrm{~d}, J=1.6 \mathrm{~Hz}, 1 \mathrm{H}) ; 7.02$ $(\mathrm{d}, J=2.0 \mathrm{~Hz}, 1 \mathrm{H}), 5.89$ (qt, $J=9.6,2.4 \mathrm{~Hz}, 2 \mathrm{H}), 5.06(\mathrm{~s}, 2 \mathrm{H}), 4.87(\mathrm{~s}, 1 \mathrm{H}), 4.85$ (d, $J=$ $5.2 \mathrm{~Hz}, 1 \mathrm{H}), 3.88(\mathrm{~s}, 3 \mathrm{H}), 3.73(\mathrm{~s}, 3 \mathrm{H}), 2.36(\mathrm{~m}, 1 \mathrm{H}), 0.78(\mathrm{~d}, J=7.2 \mathrm{~Hz}, 3 \mathrm{H}) ;{ }^{13} \mathrm{C} \mathrm{NMR}$ $\left(75 \mathrm{MHz}, \mathrm{CDCl}_{3}\right): \delta 171.3,152.2,146.8,136.21,136.20,133.9,128.6,128.1,127.3$, 123.0, 122.3, 116.8, 116.7, 73.2, 72.7, 70.8, 61.1, 52.0, 35.4, 16.0; IR (film) $v_{\max } 2956$, 1750, 1578, $1477 \mathrm{~cm}^{-1}$; HRMS(CI, Na) $\mathrm{m} / z$ calc'd for $\mathrm{C}_{22} \mathrm{H}_{24} \mathrm{BrO}_{5}[\mathrm{M}+\mathrm{H}]^{+} 447.3190$, found: 447.3184 .

(5) Wrona, I. E.; Gabarda, A. E.; Evano, G.; Panek, J. S. J. Am. Chem. Soc. 2005, 127, 15026. 
<smiles>COc1cc(Br)cc([C@@H]2O[C@H](CO)[C@@H](OC)C[C@H]2C)c1OC</smiles>

\section{[(2R,3S,5S,6S)-6-(3-Benzyloxy-5-bromo-2-methoxy-}

phenyl)-3-methoxy-5-methyl-tetrahydro-pyran-2-yl]-methanol (9b).

The dihydropyran $\mathbf{8 b}(223.1 \mathrm{mg}, 0.5 \mathrm{mmol})$ was placed in a round bottom flask equipped with $10 \mathrm{~mL}$ THF. The solution was cooled to $0{ }^{\circ} \mathrm{C}$ before borane-dimethyl sulfide complex (2.0 M in THF, $0.8 \mathrm{~mL}$ ) was added dropwise under an atmosphere of argon. The reaction mixture was allowed to warm up to room temperature and stirred for 8 hours. The mixture was cooled to $0{ }^{\circ} \mathrm{C}$ then a solution of sodium hydroxide in water $(1.0 \mathrm{M}, 5.0 \mathrm{~mL})$ and a solution of $30 \%$ aqueous hydrogen peroxide $(0.8 \mathrm{~mL})$ were added. The mixture was stirred at $0{ }^{\circ} \mathrm{C}$ for 30 minutes and at room temperature for 1 hour before $\mathrm{H}_{2} \mathrm{O}$ was added. The mixture was extracted five times with ethyl ether (aqueous phase was saturated with solid $\mathrm{NaCl}$ each time). The combined organic extracts were dried over $\mathrm{MgSO}_{4}$, filtered, and concentrated under reduced pressure to obtain the crude alcohol.

The crude oil was placed in a flame-dried $50 \mathrm{~mL}$ round bottom flask charged with 20 $\mathrm{mL}$ of dry $\mathrm{CH}_{2} \mathrm{Cl}_{2}$. Proton sponge $(535.1 \mathrm{mg}, 2.5 \mathrm{mmol})$ and $4 \AA$ molecular sieves (300 mg) were added followed by $\mathrm{Me}_{3} \mathrm{O}^{+} \mathrm{BF}_{4}^{-}(370.2 \mathrm{mg}, 2.5 \mathrm{mmol})$. The resulting heterogeneous light yellow solution was stirred for 12 hours at room temperature. The mixture was then filtered through silica and concentrated to obtain a crude oil.

The crude ester was reduced to $9 \mathbf{b}$ using general procedure (SI-5). The crude oil was purified by column chromatography over silica gel with (hexanes/ $\mathrm{CH}_{2} \mathrm{Cl}_{2} / \mathrm{Et}_{2} \mathrm{O}$ : $70 / 25 / 5$ ) to get $9 \mathrm{~b}$ as colorless oil $(126.3 \mathrm{mg}, 56 \%)$. $[\alpha]_{\mathrm{D}}^{20}+19.6\left(c 0.26, \mathrm{CHCl}_{3}\right) ;{ }^{1} \mathrm{H} \mathrm{NMR}\left(\mathrm{CDCl}_{3}\right.$, $400 \mathrm{MHz}): \delta 7.43-7.29(\mathrm{~m}, 5 \mathrm{H}), 7.22(\mathrm{~d}, J=2.0 \mathrm{~Hz}, 1 \mathrm{H}), 7.03(\mathrm{~d}, J=2.4 \mathrm{~Hz}, 1 \mathrm{H}), 5.05$ (s, 2H), 4.59 (d, $J=10.4 \mathrm{~Hz}, 1 \mathrm{H}), 4.11$ (dd, $J=10.4,4.4 \mathrm{~Hz}, 1 \mathrm{H}), 4.02$ (apt. t, $J=10.8$ Hz, 1H), 3.84 (s, 3H), 3.54 (dd, $J=11.2,4.4 \mathrm{~Hz}, 1 \mathrm{H}), 3.39$ (s, 3H), 3.23 (apt. q, $J=1.6$ $\mathrm{Hz}, 1 \mathrm{H}), 2.20(\mathrm{~m}, 1 \mathrm{H}), 2.06(\mathrm{dt}, J=14.8,2.4 \mathrm{~Hz}, 1 \mathrm{H}), 1.47$ (dd, $J=12.4,3.6 \mathrm{~Hz}, 1 \mathrm{H})$, $0.72(\mathrm{~d}, J=6.4 \mathrm{~Hz}, 3 \mathrm{H}) ;{ }^{13} \mathrm{C} \mathrm{NMR}\left(75 \mathrm{MHz}, \mathrm{CDCl}_{3}\right): \delta 152.2,147.0,136.2,135.5$, 
Mild Reductive Opening of Aryl Pyranosides Promoted by Scandium (III) Triflate

128.6, 128.2, 127.4, 122.9, 117.0, 116.7, 75.8, 74.2, 71.2, 71.0, 61.2, 59.8, 56.4, 33.2, 29.2, 17.7; IR (film) $v_{\max } 3438,2931,1576,1472 \mathrm{~cm}^{-1}$; HRMS(CI, Na) $\mathrm{m} / z$ calc'd for $\mathrm{C}_{22} \mathrm{H}_{27} \mathrm{BrNaO}_{5}[\mathrm{M}+\mathrm{Na}]^{+}$473.0940, found: 473.0768 .<smiles>COc1cc([N+](=O)[O-])c(OC)c([C@H]2O[C@H](C(C)=O)C=C[C@H]2C)c1OC</smiles>

(2S,5S,6S)-Methyl-5-methyl-6-(2,3,6-trimethoxy-5nitrophenyl)-5,6-dihydro-2H-pyran-2-carboxylate (8a).

Anti-(2R,3S,E)-crotylsilane $(385.9 \mathrm{mg}, 1.10 \mathrm{mmol})$ was placed in a round bottom flask equipped with a stir bar, $20 \mathrm{~mL} \mathrm{CH}_{2} \mathrm{Cl}_{2}$ and 2,3,6-trimethoxy-5-nitrobenzaldehyde ${ }^{4}$ (243.1 mg, $1.01 \mathrm{mmol})$. The mixture was then cooled to $-78{ }^{\circ} \mathrm{C}$ and triflic acid $(151.3$ $\mathrm{mg}, 1.0 \mathrm{mmol}$ ) was added in one portion. The reaction was allowed to stir for 24 hours at $-78{ }^{\circ} \mathrm{C}$ before a saturated solution of $\mathrm{NaHCO}_{3}$ (equal volume) was added and allowed to warm to room temperature. The aqueous layer was then extracted with additional $\mathrm{CH}_{2} \mathrm{Cl}_{2}$ (3x). The combined organic extracts were placed over $\mathrm{MgSO}_{4}$, filtered and concentrated under vacuum to get a crude oil. The resulting crude dihydropyran was purified by column chromatography over silica gel with (hexanes/ $\mathrm{CH}_{2} \mathrm{Cl}_{2} / \mathrm{EtOH}: 65 / 34 / 1$ ) to get $63 \%$ of the dihydropyran as a light yellow oil. $[\alpha]_{\mathrm{D}}^{20}-35.2\left(c 1.51, \mathrm{CHCl}_{3}\right) ;{ }^{1} \mathrm{H} \mathrm{NMR}(400$ $\left.\mathrm{MHz} \mathrm{CDCl}_{3}\right) \delta 7.45(\mathrm{~s}, 1 \mathrm{H}), 5.83(\mathrm{~m}, 2 \mathrm{H}), 4.96(\mathrm{~d}, J=9.6 \mathrm{~Hz}, 1 \mathrm{H}), 4.83$ (br s, 1H), 3.90 (s, 3H), 3.87 (s, 3H), $3.78(\mathrm{~s}, 3 \mathrm{H}), 3.72(\mathrm{~s}, 3 \mathrm{H}), 3.01(\mathrm{~m}, 1 \mathrm{H}), 0.71(\mathrm{~d}, J=7.2 \mathrm{~Hz}, 3 \mathrm{H})$; ${ }^{13} \mathrm{C} \mathrm{NMR}\left(75 \mathrm{MHz}, \mathrm{CDCl}_{3}\right) \delta 171.8,149.3,147.6,139.2,134.5,122.2,108.9,76.2,74.9$, 73.0, 72.8, 64.0, 61.8, 58.3, 52.3, 31.7, 16.7 ppm; IR (film) $v_{\max } 2951,1751,1576,1521$, $1479 \mathrm{~cm}^{-1}$; HRMS(CI, Na) $m / z$ calc'd for $\mathrm{C}_{17} \mathrm{H}_{22} \mathrm{NO}_{8}[\mathrm{M}+\mathrm{H}]^{+} 368.1345$, Found 3689.1341.<smiles>COc1cc([N+](=O)[O-])c(OC)c([C@H]2O[C@H](C(C)OC)[C@@H](OC)C[C@@H]2C)c1OC</smiles>

(2S,3S,5S,6S)-Methyl-3-methoxy-5-methyl-6-(2,3,6trimethoxy-5-nitrophenyl)-tetrahydro-2H-pyran-2-carboxylate (1c). 
The dihydropyran 8a (183.4 $\mathrm{mg}, 0.5 \mathrm{mmol})$ was placed in a round bottom flask equipped with $10 \mathrm{~mL}$ THF. The solution was cooled to $0{ }^{\circ} \mathrm{C}$ at that point borane-dimethyl sulfide complex (2.0 M in THF, $0.9 \mathrm{~mL}$ ) was added dropwise under an atmosphere of argon. The reaction mixture was allowed to warm up to room temperature and stirred for 8 hours. The mixture was cooled to $0{ }^{\circ} \mathrm{C}$ and a solution of sodium hydroxide in water $(1.0 \mathrm{M}, 5.0$ $\mathrm{mL})$ and hydrogen peroxide $(30 \% \mathrm{wt}, 1.4 \mathrm{~mL})$ were added. The mixture was stirred at 0 ${ }^{\circ} \mathrm{C}$ for 30 minutes and at room temperature for 1 hour before $\mathrm{H}_{2} \mathrm{O}$ was added to dilute. The mixture was extracted with $\mathrm{Et}_{2} \mathrm{O}(5 \mathrm{x}$, aqueous phase was saturated with solid $\mathrm{NaCl}$ each time). The combined organic extracts were dried over $\mathrm{MgSO}_{4}$, filtered, and concentrated under reduced pressure to get crude alcohol.

The crude oil was placed in flame-dried $25 \mathrm{~mL}$ round bottom flask $20 \mathrm{~mL}$ of dry $\mathrm{CH}_{2} \mathrm{Cl}_{2}$, proton sponge $(535.1 \mathrm{mg}, 2.5 \mathrm{mmol})$ and $4 \AA$ molecular sieves $(300 \mathrm{mg})$ were added followed by $\mathrm{Me}_{3} \mathrm{O}^{+} \mathrm{BF}_{4}^{-}(370.2 \mathrm{mg}, 2.5 \mathrm{mmol})$. The resulting heterogeneous light yellow solution was stirred for 12 hours at room temperature. The mixture was then filtered through silica and concentrated to obtain a crude oil. The crude oil was purified by column chromatography over silica gel with (hexanes $/ \mathrm{CH}_{2} \mathrm{Cl}_{2} / \mathrm{Et}_{2} \mathrm{O}: 80 / 15 / 5$ ) to get $68 \%$ yield of the 3-methoxyl tetrahydropyran as yellow oil (135.6 $\mathrm{mg}, 0.34 \mathrm{mmol}$ ). $[\alpha]_{\mathrm{D}}^{20}+9.9\left(c 2.70, \mathrm{CHCl}_{3}\right) ;{ }^{1} \mathrm{H} \mathrm{NMR}\left(400 \mathrm{MHz}, \mathrm{CDCl}_{3}\right) \delta 7.40(\mathrm{~s}, 1 \mathrm{H}), 4.99(\mathrm{~d}, J=3.8$ $\mathrm{Hz}, 1 \mathrm{H}), 4.61$ (s, 1H), 3.91(s, 3H), 3.83 (s, 3H), 3.81 (m, 1H), 3.76 (s, 3H), 3.72(s, 3H), $3.42(\mathrm{~s}, 3 \mathrm{H}), 2.95(\mathrm{~m}, 1 \mathrm{H}), 2.05(\mathrm{~m}, 1 \mathrm{H}), 1.22(\mathrm{~m}, 1 \mathrm{H}), 0.56(\mathrm{~d}, J=6.2 \mathrm{~Hz}, 3 \mathrm{H}) ;{ }^{13} \mathrm{C}$ NMR (75 MHz, $\left.\mathrm{CDCl}_{3}\right) \delta 171.6,152.3,148.7,130.5,108.7,107.8,81.0,78.1,74.9,73.7$, 63.7, 61.8, 59.3, 56.3, 52.3, 33.6, 25.8, 17.8 ppm; IR (film) $v_{\max } 2942,1746,1521,1475$, $1336 \mathrm{~cm}^{-1}$; HRMS(CI, Na) $\mathrm{m} / z$ calc'd for $\mathrm{C}_{18} \mathrm{H}_{25} \mathrm{NNaO}_{9}[\mathrm{M}+\mathrm{Na}]^{+}$422.1427, found: 422.1425. 
<smiles>COc1cc([N+](=O)[O-])c(OC)c([C@H]2O[C@H](CO)[C@@H](OC)C[C@H]2C)c1OC</smiles>

[(2R,3S,5S,6S)-3-Methoxy-5-methyl-6-(2,3,6-trimethoxy-5nitrophenyl)-tetrahydro-2H-pyran-2-yl]methanol (9a). (See the general procedure for reduction of methyl ester to alcohol on SI-5 to obtain $97 \%$ reduced from ester 1c as a light yellow oil); $[\alpha]_{\mathrm{D}}^{20}+3.1^{\circ}\left(c 0.84, \mathrm{CHCl}_{3}\right) ;{ }^{1} \mathrm{H} \mathrm{NMR}\left(400 \mathrm{MHz}, \mathrm{CDCl}_{3}\right) \delta 7.36(\mathrm{~s}$, 1H), 5.01 (d, $J=3.8 \mathrm{~Hz}, 1 \mathrm{H}), 4.61$ (br s, 1H), 3.89 (s, 3H), 3.87 (s, 3H), $3.81(\mathrm{~m}, 1 \mathrm{H})$, $3.79(\mathrm{~s}, 3 \mathrm{H}), 3.44(\mathrm{~m}, 2 \mathrm{H}), 3.41(\mathrm{~s}, 3 \mathrm{H}), 2.93(\mathrm{~m}, 1 \mathrm{H}), 2.20$ (br s, 1H), $2.05(\mathrm{~m}, 1 \mathrm{H}), 1.24$ $(\mathrm{m}, 1 \mathrm{H}), 0.62(\mathrm{~d}, J=7.2 \mathrm{~Hz}, 3 \mathrm{H}) ;{ }^{13} \mathrm{C} \mathrm{NMR}\left(75 \mathrm{MHz}, \mathrm{CDCl}_{3}\right) \delta 152.5,148.9,130.2$, 107.7, 107.5, 81.0, 78.1, 73.6, 71.7, 63.7, 61.5, 59.3, 56.4, 36.2, 34.5, 25.819 .5 ppm; IR (film) $v_{\max }$ 3467, 2952, 1521, $1475 \mathrm{~cm}^{-1}$; HRMS(CI, Na) $\mathrm{m} / z$ calc'd for $\mathrm{C}_{17} \mathrm{H}_{25} \mathrm{NO}_{8}[\mathrm{M}]^{+}$371.1580, Found 371.1582.<smiles>[M]Oc1cc(OC)c([C@H]2O[C@H](COC)[C@@H](OC)[C@H](OC)[C@H]2OC)c(OC)c1</smiles>

$(2 R, 3 R, 4 R, 5 S, 6 S)-3,4,5-T$ rimethoxy-2-methoxymethyl-6$\left(2,4,6\right.$-trimethoxy-phenyl)-tetrahydro-pyran (11). (Prepared from the alkylation ${ }^{6}$ of 2,4,5-trimethoxy- $\beta$-D-glucopyranoside ${ }^{7}$ in $75 \%$ yield $) .[\alpha]_{\mathrm{D}}^{20}-2.5$ (c $\left.0.44, \mathrm{CHCl}_{3}\right) ;{ }^{1} \mathrm{H}$ NMR $\left(\mathrm{CDCl}_{3}, 400 \mathrm{MHz}\right): \delta 6.08(\mathrm{~s}, 2 \mathrm{H}), 4.68(\mathrm{~d}, J=10 \mathrm{~Hz}, 1 \mathrm{H}), 3.95(\mathrm{~m}, 1 \mathrm{H}), 3.77(\mathrm{~s}$, 3H), 3.76 (s, 3H), 3.75 (s, 3H), 3.65 (s, 3H), 3.63-3.54 (obsc. m, 3H), 3.56 (s, 3H), 3.35 (s, 3H), 3.25-3.18 (m, 2H), $3.11(\mathrm{~s}, 3 \mathrm{H}) ;{ }^{13} \mathrm{C}$ NMR (75 MHz, $\left.\mathrm{CDCl}_{3}\right): \delta 161.0,160.7$, 159.7, 107.3, 91.5, 90.6, 88.9, 81.1, 79.8, 79.0, 72.3, 71.6, 60.6, 60.2, 59.4, 59.1, 56.0, 55.5, 55.0; IR (film) $v_{\max }$ 2937, 2835, 1603, 1461, $1202 \mathrm{~cm}^{-1} ; \operatorname{HRMS}(\mathrm{CI}, \mathrm{Na}) \mathrm{m} / \mathrm{z}$ calc'd for $\mathrm{C}_{19} \mathrm{H}_{30} \mathrm{NaO}_{8}[\mathrm{M}+\mathrm{Na}]^{+} 409.1838$, found: 409.1806 .

(6) Wang, H.; Sun, L.; Glazebnik, S.; Zhao, K. Tetrahedron Lett. 1995, 36, 2953.

(7) For the preparation of the polyol see: Stewart, A. O.; Williams, R. M. J. Am. Chem. Soc. 1985, 107, 4289. 


\section{General procedure for reductive opening of 5-aryl pyrans}<smiles>[R2][C@@H]1O[C@H]([AlH])[C@@H](C)C[C@H]1[R7]</smiles><smiles></smiles><smiles>[R]C(O)C([R])C[C@@H](C)C[3H]</smiles>

Condition $\boldsymbol{A}$ (Room temperature):

Triethyl silane (5 equiv.) was placed in a round bottom flask containing a stir bar and a 0.1-0.5M solution of the appropriate pyran $(0.1 \mathrm{mmol})$ in $\mathrm{CH}_{2} \mathrm{Cl}_{2}$. To this solution $\mathrm{Sc}(\mathrm{OTf})_{3}$ (2.0 equiv.) was added and allowed to stir for 24 hours at room temperature. Water was then added and the mixture was allowed to stir for 10 minutes before being extracted with $\mathrm{CH}_{2} \mathrm{Cl}_{2}(3 \mathrm{x})$. The combined organic extracts were dried over $\mathrm{MgSO}_{4}$, filtered, and concentrated under reduced pressure. The residue was purified by flash chromatography over silica gel to yield the desired alcohol. The aqueous layer was washed with $\mathrm{CH}_{2} \mathrm{Cl}_{2}$ and concentrated under reduced pressure to recover $\mathrm{Sc}(\mathrm{OTf})_{3}$.

\section{Condition B (Microwave):}

Triethyl silane (5 equiv.) was placed in a microwave tube containing a stir bar and a 0.1 $0.5 \mathrm{M}$ solution of the appropriate pyrans $(0.1 \mathrm{mmol})$ in $\mathrm{CH}_{2} \mathrm{Cl}_{2}$ at ambient temperature. To the solution $\mathrm{Sc}(\mathrm{OTf})_{3}(0.25-1.0$ equiv.) was then added. The tube was sealed and irradiated in a microwave (300 watts, 70 PSI and $100{ }^{\circ} \mathrm{C}$ ) for 20 to 60 minutes before water was added. The mixture was allowed to stir for 10 minutes then extracted with additional $\mathrm{CH}_{2} \mathrm{Cl}_{2}(3 \mathrm{x})$. The combined organic extracts were dried over $\mathrm{MgSO}_{4}$, filtered, and concentrated under reduced pressure. The residue was purified by flash chromatography over silica gel to yield the desired alcohol. The aqueous solution was washed with $\mathrm{CH}_{2} \mathrm{Cl}_{2}$ and concentrated under reduced pressure to recover $\mathrm{Sc}(\mathrm{OTf})_{3}$.<smiles>CC(=O)[C@H](O)CC[C@H](C)Cc1ccccc1</smiles>

(2S,5S)-Methyl-2-hydroxy-5-methyl-6-phenylhexanoate

(2a). (Condition A (60 hours) provided 2a from 1a in 67\% overall yield as colorless oil; Condition B (2.0 equiv. Sc(OTf $)_{3}$ ) provided 2a from 1a in $71 \%$ overall yield as colorless 
Mild Reductive Opening of Aryl Pyranosides Promoted by Scandium (III) Triflate

oil ); $[\alpha]_{\mathrm{D}}^{20}+1.7\left(\right.$ c $\left.2.20, \mathrm{CHCl}_{3}\right) ;{ }^{1} \mathrm{H}$ NMR $\left(400 \mathrm{MHz}, \mathrm{CDCl}_{3}\right) \delta$ 7.14-7.22 (m, 5H), 4.17 (dd, $J=5.8,2.8 \mathrm{~Hz}, 1 \mathrm{H}), 3.74(\mathrm{~s}, 3 \mathrm{H}), 2.69$ (s, 1H), 2.61 (q, $J=6.0 \mathrm{~Hz}, 1 \mathrm{H}), 2.38$ $(\mathrm{q}, J=5.8 \mathrm{~Hz}, 1 \mathrm{H}), 1.77(\mathrm{~m}, 3 \mathrm{H}), 1.49(\mathrm{~m}, 1 \mathrm{H}), 1.21(\mathrm{~m}, 1 \mathrm{H}), 0.82(\mathrm{~d}, J=6.4 \mathrm{~Hz}, 3 \mathrm{H})$; ${ }^{13} \mathrm{C}$ NMR $\left(75 \mathrm{MHz}, \mathrm{CDCl}_{3}\right) \delta 175.9,141.4,129.4,128.4,126.0,70.9,52.7,43.7,35.0$, 32.2, 31.7, 19.4 ppm; IR (film) $v_{\max } 3466,3025,2953,1734,1495,1453 \mathrm{~cm}^{-1}$; HRMS(CI, Na) $m / z$ calc'd for $\mathrm{C}_{14} \mathrm{H}_{20} \mathrm{O}_{3}[\mathrm{M}]^{+}$236.1412, Found 236.1407 .

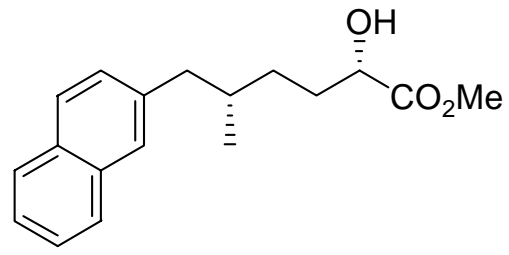

(2S,5S)-Methyl-2-hydroxy-5-methyl-6-(naphthalen-2-

yl)-hexanoate (2b). (Condition A provided $\mathbf{2 b}$ from $\mathbf{1 b}$ in $83 \%$ overall yield as colorless oil; Condition B (2.0equiv. Sc(OTf $)_{3}$ ) provided $\mathbf{2 b}$ from $\mathbf{1 b}$ in $86 \%$ overall yield as colorless oil); $[\alpha]_{\mathrm{D}}^{20}+22.3\left(\right.$ c $\left.2.41, \mathrm{CHCl}_{3}\right) ;{ }^{1} \mathrm{H} \mathrm{NMR}\left(400 \mathrm{MHz}, \mathrm{CDCl}_{3}\right) \delta 7.74(\mathrm{~m}, 3 \mathrm{H})$, $7.55(\mathrm{~s}, 1 \mathrm{H}), 7.40(\mathrm{~m}, 2 \mathrm{H}), 7.26(\mathrm{~d}, J=8.0 \mathrm{~Hz}, 1 \mathrm{H}), 4.18(\mathrm{dd}, J=7.1,3.2 \mathrm{~Hz}, 1 \mathrm{H}), 3.71$ (s, 3H), 2.69 (q, $J=5.8 \mathrm{~Hz}, 1 \mathrm{H}), 2.57$ (q, $J=5.9 \mathrm{~Hz}, 1 \mathrm{H}), 2.22(\mathrm{br} \mathrm{s}, 1 \mathrm{H}), 1.79(\mathrm{~m}, 3 \mathrm{H})$, $1.48(\mathrm{~m}, 1 \mathrm{H}), 1.21(\mathrm{~m}, 1 \mathrm{H}), 0.87(\mathrm{~d}, J=8.0 \mathrm{~Hz}, 3 \mathrm{H}) ;{ }^{13} \mathrm{C} \mathrm{NMR}\left(75 \mathrm{MHz}, \mathrm{CDCl}_{3}\right) \delta$ 175.9, 138.9, 133.7, 132.2, 128.1, 127.9, 127.8, 127.6, 127.5, 126.0, 125.3, 70.8, 52.7, 43.9, 35.0, 32.2, 31.8, 19.5 ppm; IR (film) $v_{\max } 3471,3052$, 2952, 1734, 1599, 1507, $1437 \mathrm{~cm}^{-1}$; HRMS(CI, Na) $\mathrm{m} / z$ calc'd for $\mathrm{C}_{18} \mathrm{H}_{22} \mathrm{O}_{3}[\mathrm{M}]^{+}$286.1569, Found 286.1562 .<smiles>COc1cc([N+](=O)[O-])c(OC)c(C[C@@H](C)C[C@H](OC)[C@H](O)C(C)=O)c1OC</smiles>

(2S,3S,5R)-Methyl-2-hydroxy-3-methoxy-5-methyl6-(2,3,6-trimethoxy-5-nitrophenyl)-hexanoate ${ }^{3}$ (2c). (Condition A provided $2 \mathrm{c}$ from 1c in $82 \%$ overall yield as light yellow oil); $[\alpha]_{\mathrm{D}}^{20}+0.3\left(\right.$ c $\left.2.70, \mathrm{CHCl}_{3}\right) ;{ }^{1} \mathrm{H} \mathrm{NMR}(400 \mathrm{MHz}$, $\left.\mathrm{CDCl}_{3}\right) \delta 7.35(\mathrm{~s}, 1 \mathrm{H}), 4.42(\mathrm{dd}, J=5.5,3.0 \mathrm{~Hz}, 1 \mathrm{H}), 3.88(\mathrm{~s}, 3 \mathrm{H}), 3.86(\mathrm{~s}, 3 \mathrm{H}), 3.84(\mathrm{~s}$, $3 \mathrm{H}), 3.78(\mathrm{~s}, 3 \mathrm{H}), 3.52(\mathrm{dt}, J=9.7,3.0 \mathrm{~Hz}, 1 \mathrm{H}), 3.38(\mathrm{~s}, 3 \mathrm{H}), 2.88(\mathrm{~d}, J=5.7 \mathrm{~Hz}, 1 \mathrm{H})$, 
Mild Reductive Opening of Aryl Pyranosides Promoted by Scandium (III) Triflate

$2.63(\mathrm{dd}, J=12.4,6.2 \mathrm{~Hz}, 1 \mathrm{H}), 2.53(\mathrm{dd}, J=12.9,8.7 \mathrm{~Hz}, 1 \mathrm{H}), 2.09-1.96(\mathrm{~m}, 1 \mathrm{H}), 1.75$ $(\mathrm{m}, 1 \mathrm{H}), 1.17(\mathrm{~m}, 1 \mathrm{H}), 0.81(\mathrm{~d}, J=6.8 \mathrm{~Hz}, 3 \mathrm{H}) ;{ }^{13} \mathrm{C} \mathrm{NMR}\left(75 \mathrm{MHz}, \mathrm{CDCl}_{3}\right) \delta 173.3$, 152.8, 148.4, 148.0, 138.3, 131.1, 107.1, 81.0, 71.9, 62.6, 61.1, 58.3, 56.3, 52.7, 37.3, 32.2, 30.2, 19.5 ppm; IR (film) $v_{\max } 3498,2952,1736,1518,1479,1424 \mathrm{~cm}^{-1} \operatorname{HRMS(CI,~}$ Na) $\mathrm{m} / z$ calc'd for $\mathrm{C}_{18} \mathrm{H}_{27} \mathrm{NNaO}_{9} 424.1584$, Found 424.1588 . Found to be identical to previous reports of $\mathbf{2}$. $^{4}$

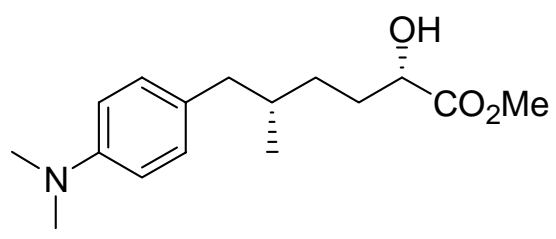

(2S,5S)-Methyl-6-(4-(dimethylamino)phenyl)-2-

hydroxy-5-methylhexanoate (2d). (Condition A provided 2d from 1d in $73 \%$ overall yield as light yellow oil); $[\alpha]_{\mathrm{D}}^{20}+2.7\left(c 2.01, \mathrm{CHCl}_{3}\right) ;{ }^{1} \mathrm{H} \mathrm{NMR}\left(400 \mathrm{MHz}, \mathrm{CDCl}_{3}\right) \delta$ $7.01(\mathrm{~d}, J=8.8 \mathrm{~Hz}, 2 \mathrm{H}), 6.68(\mathrm{~d}, J=8.8 \mathrm{~Hz}, 2 \mathrm{H}), 4.16(\mathrm{~m}, 1 \mathrm{H}), 3.75(\mathrm{~s}, 3 \mathrm{H}), 2.91(\mathrm{~s}$, $6 \mathrm{H}), 2.67(\mathrm{~s}, 1 \mathrm{H}), 2.53(\mathrm{dd}, J=16.2,4.8 \mathrm{~Hz}, 1 \mathrm{H}), 2.26(\mathrm{dd}, J=16.2,4.8 \mathrm{~Hz}, 1 \mathrm{H}), 1.67$ $(\mathrm{m}, 3 \mathrm{H}), 1.50(\mathrm{~m}, 1 \mathrm{H}), 1.18(\mathrm{~m}, 1 \mathrm{H}), 0.82(\mathrm{~d}, J=6.8 \mathrm{~Hz}, 3 \mathrm{H}) ;{ }^{13} \mathrm{C} \mathrm{NMR}(75 \mathrm{MHz}$, $\left.\mathrm{CDCl}_{3}\right) \delta 175.7,140.0,139.6,130.3,122.4,70.5,52.5,42.9,34.8,32.2,32.0,31.5,19.1$ ppm; IR (film) $v_{\max } 3474,2952,2801,1739 \mathrm{~cm}^{-1}$; HRMS(CI, Na) $\mathrm{m} / z$ calc'd for $\mathrm{C}_{16} \mathrm{H}_{26} \mathrm{NO}_{3}[\mathrm{M}]^{+}$280.1913, Found 280.1917.<smiles>COc1cc(Br)c(OC)c(C[C@@H](C)CC(OC)C(O)C(C)=O)c1OC</smiles>

trimethoxyphenyl)-2-hydroxy-3-methoxy-5-methylhexanoate (2e). (Condition A provided $2 \mathbf{e}$ from $1 \mathbf{e}$ in $87 \%$ overall yield as colorless oil in $2.5 \mathrm{~g}$; Condition $\mathbf{B}$ (2.0 equiv. $\left.\mathrm{Sc}(\mathrm{OTf})_{3}\right)$ provided $2 \mathbf{e}$ from 1e in $89 \%$ overall yield as colorless oil $) ;[\alpha]_{\mathrm{D}}^{20}+3.7^{\circ}(c 2.20$, $\left.\mathrm{CHCl}_{3}\right) ;{ }^{1} \mathrm{H} \mathrm{NMR}\left(400 \mathrm{MHz}, \mathrm{CDCl}_{3}\right) \delta 6.92(\mathrm{~s}, 1 \mathrm{H}), 4.43(\mathrm{~m}, 1 \mathrm{H}), 3.81(\mathrm{~s}, 3 \mathrm{H}), 3.79$ (s, $3 \mathrm{H}), 3.77(\mathrm{~s}, 3 \mathrm{H}), 3.74(\mathrm{~s}, 3 \mathrm{H}), 3.55(\mathrm{~m}, 1 \mathrm{H}), 3.40(\mathrm{~s}, 3 \mathrm{H}), 2.87(\mathrm{~d}, 1 \mathrm{H}), 2.61(\mathrm{dd}, J=$ $16.2,3.8 \mathrm{~Hz}, 1 \mathrm{H}), 2.45(\mathrm{dd},, J=16.2,3.8 \mathrm{~Hz}, 1 \mathrm{H}), 2.02(\mathrm{~m}, 1 \mathrm{H}), 1.71(\mathrm{~m}, 1 \mathrm{H}), 1.12(\mathrm{~m}$, $1 \mathrm{H}), 0.80(\mathrm{~d}, J=9.2 \mathrm{~Hz}, 3 \mathrm{H}) ;{ }^{13} \mathrm{C} \mathrm{NMR}\left(75 \mathrm{MHz}, \mathrm{CDCl}_{3}\right) \delta 173.5,150.0,147.7,130.2$, 
Mild Reductive Opening of Aryl Pyranosides Promoted by Scandium (III) Triflate

$114.3,110.8,81.2,72.2,61.3,60.8,58.4,56.3,52.7,37.5,33.0,32.0,30.6,19.6$ ppm; IR (film) $v_{\max } 3469,2933,1739,1475 \mathrm{~cm}^{-1}$; $\mathrm{HRMS}(\mathrm{CI}, \mathrm{Na}) \mathrm{m} / z$ calc'd for $\mathrm{C}_{18} \mathrm{H}_{27} \mathrm{BrO}_{7}[\mathrm{M}]^{+}$ 434.0940, Found 434.0941.

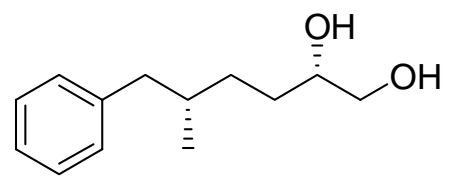

(2S,5S)-5-methyl-6-phenylhexane-1,2-diol (2f). (Condition A provided $2 \mathbf{f}$ from 1 if in $78 \%$ overall yield as colorless oil; Condition B (2.0 equiv. $\left.\mathrm{Sc}(\mathrm{OTf})_{3}\right)$ provided $\mathbf{2 f}$ from $\mathbf{1 f}$ in $71 \%$ overall yield as colorless oil $) ;[\alpha]_{\mathrm{D}}^{20}+33.4$ (c 2.90, $\left.\mathrm{CHCl}_{3}\right) ;{ }^{1} \mathrm{H}$ NMR $\left(400 \mathrm{MHz}, \mathrm{CDCl}_{3}\right) \delta 7.25(\mathrm{~m}, 5 \mathrm{H}), 3.62(\mathrm{~m}, 2 \mathrm{H}), 3.42(\mathrm{~m}, 1 \mathrm{H}), 2.62$ (dd, $J=13.6,4.2 \mathrm{~Hz}, 1 \mathrm{H}), 2.37$ (dd, $J=13.6,4.2 \mathrm{~Hz}, 1 \mathrm{H}), 1.96$ (s, 1H), 1.69 (m, 2H), $1.48(\mathrm{~m}, 3 \mathrm{H}), 0.85(\mathrm{~d}, J=6.8 \mathrm{~Hz}, 3 \mathrm{H}) ;{ }^{13} \mathrm{C} \mathrm{NMR}\left(75 \mathrm{MHz}, \mathrm{CDCl}_{3}\right) \quad \delta 141.3,128.6$, 128.1, 127.6, 79.3, 73.2, 61.6, 35.4, 27.7, 25.1, 18.4 ppm; IR (film) $v_{\max } 3352,3026$, 2925, 160, 1494, $1454 \mathrm{~cm}^{-1} ; \operatorname{HRMS}(\mathrm{CI}, \mathrm{Na}) \mathrm{m} / z$ calc'd for $\mathrm{C}_{13} \mathrm{H}_{20} \mathrm{O}_{2}[\mathrm{M}+\mathrm{Na}]^{+}$ 231.1361, Found 231.1359.<smiles>C[C@H](CC[C@H](O)CO)Cc1cccc2ccccc12</smiles>

(2S,5S)-5-Methyl-6-(naphthalen-1-yl)hexane-1,2-diol (2g). (Condition A provided $\mathbf{2 g}$ from $\mathbf{1 g}$ in $93 \%$ overall yield as colorless oil; Condition B (1.0 equiv. $\left.\mathrm{Sc}(\mathrm{OTf})_{3}\right)$ provided $\mathbf{2 g}$ from $\mathbf{1 g}$ in $89 \%$ overall yield as colorless oil) $[\alpha]_{\mathrm{D}}^{20}+$ $60.2\left(c 4.48, \mathrm{CHCl}_{3}\right) ;{ }^{1} \mathrm{H} \mathrm{NMR}\left(400 \mathrm{MHz}, \mathrm{CDCl}_{3}\right) \delta 7.98(\mathrm{~d}, J=8.6 \mathrm{~Hz}, 1 \mathrm{H}), 7.84(\mathrm{~d}, J$ $=8.6 \mathrm{~Hz}, 1 \mathrm{H}), 7.70(\mathrm{~d}, J=9.0 \mathrm{~Hz}, 1 \mathrm{H}), 7.46(\mathrm{~m}, 2 \mathrm{H}), 7.36(\mathrm{~m}, 1 \mathrm{H}), 7.25(\mathrm{~d}, J=6.4 \mathrm{~Hz}$, $1 \mathrm{H}), 3.63(\mathrm{~m}, 2 \mathrm{H}), 3.40(\mathrm{~m}, 1 \mathrm{H}), 3.11(\mathrm{dd}, J=17.8,3.8 \mathrm{~Hz}, 1 \mathrm{H}), 2.79(\mathrm{dd}, J=17.8,4.6$ $\mathrm{Hz}, 1 \mathrm{H}), 2.12(\mathrm{~s}, 1 \mathrm{H}), 1.91(\mathrm{~m}, 2 \mathrm{H}), 1.55(\mathrm{~m}, 1 \mathrm{H}), 1.43(\mathrm{~m}, 2 \mathrm{H}), 0.90(\mathrm{~d}, J=6.4 \mathrm{~Hz}$, $3 \mathrm{H}) ;{ }^{13} \mathrm{C} \mathrm{NMR}\left(75 \mathrm{MHz}, \mathrm{CDCl}_{3}\right) \delta 137.5,134.1,132.4,129.0,127.5,126.9,125.8$, 125.6, 125.5, 124.2, 72.8, 67.0, 41.1, 34.4, 33.1, 30.9, 19.9 ppm; IR (film) $v_{\max } 3367$, 3064, 2926, 1595, 1509, $1458 \mathrm{~cm}^{-1}$; $\operatorname{HRMS}(\mathrm{CI}, \mathrm{Na}) \mathrm{m} / z$ calc'd for $\mathrm{C}_{17} \mathrm{H}_{22} \mathrm{O}_{2}[\mathrm{M}]^{+}$ 258.1620, Found 258.1620. 
<smiles>COc1cc(Br)c(OC)c(C[C@@H](C)C[C@H](OC)[C@H](O)CO)c1OC</smiles>

(2R,3S,5R)-6-(3-Bromo-2,5,6-trimethoxyphenyl)-3-

methoxy-5-methylhexane-1,2-diol (2h). (Condition A provided $\mathbf{2 h}$ from $\mathbf{1 h}$ in $88 \%$ overall yield as colorless oil; Condition $\mathbf{B}\left(0.25\right.$ equiv. $\left.\mathrm{Sc}(\mathrm{OTf})_{3}\right)$ provided $\mathbf{2 h}$ from $\mathbf{1 h}$ in $80 \%$ overall yield as colorless oil); $[\alpha]_{\mathrm{D}}^{20}-4.8$ (c 2.20, $\left.\mathrm{CHCl}_{3}\right) ;{ }^{1} \mathrm{H}$ NMR $(400 \mathrm{MHz}$, $\left.\mathrm{CDCl}_{3}\right) \delta 6.89(\mathrm{~s}, 1 \mathrm{H}), 3.76(\mathrm{~s}, 3 \mathrm{H}), 3.74(\mathrm{~s}, 3 \mathrm{H}), 3.71(\mathrm{~s}, 3 \mathrm{H}), 3.58(\mathrm{~m}, 2 \mathrm{H}), 3.33(\mathrm{~s}$, $3 \mathrm{H}), 3.18(\mathrm{~m}, 1 \mathrm{H}), 2.95(\mathrm{~m}, 1 \mathrm{H}), 2.58(\mathrm{dd}, J=13.6,3.6 \mathrm{~Hz}, 1 \mathrm{H}), 2.43(\mathrm{dd}, J=13.6,4.2$ $\mathrm{Hz}, 1 \mathrm{H}), 2.18$ (br s, 2H), $1.99(\mathrm{~m}, 1 \mathrm{H}), 1.59$ (m, 1H), $1.22(\mathrm{~m}, 1 \mathrm{H}), 0.82$ (d, J = $6.8 \mathrm{~Hz}$, $3 \mathrm{H}) ;{ }^{13} \mathrm{C} \mathrm{NMR}\left(75 \mathrm{MHz}, \mathrm{CDCl}_{3}\right) \delta$ 150.1, 149.7, 130.1, 114.3, 110.6, 90.1, 73.1, 63.4, 61.2, 60.9, 60.6, 58.2, 56.1, 37.9, 33.0, 30.4, 19.7 ppm; IR (film) v $v_{\max } 3450,2938,1475$, $1425 \mathrm{~cm}^{-1}$; HRMS(CI, Na) $\mathrm{m} / z$ calc'd for $\mathrm{C}_{17} \mathrm{H}_{27} \mathrm{BrNaO}_{6}[\mathrm{M}+\mathrm{Na}]^{+} 429.0889$, Found 429.0885 .<smiles>COc1c(C)c(OC)c(OC)c(C[C@@H](C)CC[C@H](O)C(C)=O)c1OC</smiles>

(2S,5R)-2-Hydroxy-5-methyl-6-(2,3,5,6tetramethoxy-4-methyl-phenyl)-hexanoic acid methyl ester (2i). (Condition $A$ provided $2 \mathbf{i}$ from $1 \mathbf{i}$ in $78 \%$ overall yield as colorless oil). ${ }^{3}[\alpha]_{\mathrm{D}}^{20}-16.9\left(c 0.82, \mathrm{CHCl}_{3}\right)$; ${ }^{1} \mathrm{H}$ NMR $\left(\mathrm{CDCl}_{3}, 400 \mathrm{MHz}\right): \delta 4.19(\mathrm{~m}, 1 \mathrm{H}), 3.78$ (s, 3H), 3.75 (s, 12H), 2.70 (br. s, $\left.1 \mathrm{H}\right)$, $2.56(\mathrm{dd}, J=16.8,8.0 \mathrm{~Hz}, 1 \mathrm{H}), 2.38(\mathrm{dd}, J=16.8,11.6 \mathrm{~Hz}, 1 \mathrm{H}), 2.14$ (s, 3H), 1.89 (m, 1H), $1.73(\mathrm{~m}, 1 \mathrm{H}), 1.64(\mathrm{~m}, 1 \mathrm{H}), 1.37(\mathrm{~m}, 2 \mathrm{H}), 0.84(\mathrm{~d}, J=9.2 \mathrm{~Hz}, 3 \mathrm{H}) ;{ }^{13} \mathrm{C}$ NMR $(75$ $\left.\mathrm{MHz}_{\mathrm{CDCl}}\right): \delta 175.8,147.7,147.3,125.9,123.3,70.7,60.4,60.1,52.3,33.7,32.0$, 31.9, 31.1, 19.7, 9.1; IR (film) $v_{\max } 3478,2951,1740,1462,1406 \mathrm{~cm}^{-1}$; HRMS(CI, Na) $m / z$ calc'd for $\mathrm{C}_{19} \mathrm{H}_{30} \mathrm{NaO}_{7}[\mathrm{M}+\mathrm{Na}]^{+} 393.1889$, found: 393.1910 . 
<smiles>COc1c(C)c(OC)c(OC)c(C[C@@H](C)[C@H](O)[C@H](C)[C@H](O)CO)c1OC</smiles>

$(2 S, 3 R, 4 R, 5 S)-3,5-D i m e t h y l-6-(2,3,5,6-t e t r a m e t h o x y-4-$ methyl-phenyl)-hexane-1,2,4-triol (2j). (Condition $\boldsymbol{A}$ provided $\mathbf{2 j}$ from $\mathbf{1 j}$ in $62 \%$ overall yield as colorless oil); $[\alpha]_{\mathrm{D}}^{20}+65.5\left(\right.$ c 1.02, $\left.\mathrm{CHCl}_{3}\right) ;{ }^{1} \mathrm{H} \mathrm{NMR}\left(\mathrm{CDCl}_{3}, 400 \mathrm{MHz}\right)$ : $\delta 3.81(\mathrm{~s}, 6 \mathrm{H}), 3.75(\mathrm{~s}, 6 \mathrm{H}), 3.63(\mathrm{~s}, 1 \mathrm{H}), 3.48$ (s, 2H), 3.29 (br. s, 2H), 3.17 (d, J= 10.8 $\mathrm{Hz}, 1 \mathrm{H}), 2.65(\mathrm{dd}, J=13.2,4.4 \mathrm{~Hz}, 1 \mathrm{H}), 2.46(\mathrm{t}, J=12.4 \mathrm{~Hz}, 1 \mathrm{H}), 2.15(\mathrm{~s}, 3 \mathrm{H}), 2.00$ (m, $1 \mathrm{H}), 1.76(\mathrm{~m}, 1 \mathrm{H}), 0.97(\mathrm{~d}, J=6.8 \mathrm{~Hz}, 3 \mathrm{H}), 0.67(\mathrm{~d}, J=6.8 \mathrm{~Hz}, 3 \mathrm{H}) ;{ }^{13} \mathrm{C} \mathrm{NMR}(75 \mathrm{MHz}$, $\left.\mathrm{CDCl}_{3}\right): \delta 147.7,147.3,124.4,124.1,73.7,73.4,63.5,60.9,60.3,37.3,35.9,28.4,13.3$, 12.8, 9.1; IR (film) $v_{\max } 3425,2936,1462,1406,1077 \mathrm{~cm}^{-1}$; HRMS(CI, Na) $m / z$ calc'd for $\mathrm{C}_{19} \mathrm{H}_{33} \mathrm{O}_{7}[\mathrm{M}+\mathrm{H}]^{+}$373.2226, found: 373.2221.<smiles>OC[C@@H](O)CCc1ccccc1</smiles>

(2R,3S)-5-Phenyl-pentane-1,2,3-triol (2k). (Condition A (48 hours) provided $\mathbf{2 k}$ from $\mathbf{1 k}$ in $50 \%$ overall yield from 1-phenyl-2-deoxy-D-ribose ${ }^{8}$ as colorless oil; Condition B (2.0 equiv. $\left.\mathrm{Sc}(\mathrm{OTf})_{3}\right)$ provided $\mathbf{2 k}$ from $\mathbf{1 k}$ in $51 \%$ overall yield from 1-phenyl-2-deoxy-D-ribose as colorless oil); $[\alpha]_{\mathrm{D}}^{20}-26.0\left(c 0.15, \mathrm{CHCl}_{3}\right) ;{ }^{1} \mathrm{H}$ NMR (CD $\left.\mathrm{CD}_{3} \mathrm{OD} 4 \mathrm{MHz}\right): \delta$ 7.25-7.19 (m, 4H), 7.14-7.11 (m, 1H), $3.72(\mathrm{dd}, J=12.4$, $4.0 \mathrm{~Hz}, 1 \mathrm{H}), 3.56(\mathrm{~d}, J=6.8 \mathrm{~Hz}, 1 \mathrm{H}), 3.54-3.42(\mathrm{~m}, 2 \mathrm{H}), 2.85(\mathrm{~m}, 1 \mathrm{H}), 2.63(\mathrm{~m}, 1 \mathrm{H})$, $1.97(\mathrm{~m}, 1 \mathrm{H}), 1.68(\mathrm{~m}, 1 \mathrm{H}) ;{ }^{13} \mathrm{C}$ NMR (75 MHz, $\left.\mathrm{CD}_{3} \mathrm{OD}\right): \delta 143.7,129.5,129.3,126.7$, 76.3, 72.8, 64.7, 36.3, 32.9; IR (film) $v_{\max } 3329,2919,1454,1041 \mathrm{~cm}^{-1}$; HRMS(CI, Na) $m / z$ calc'd for $\mathrm{C}_{11} \mathrm{H}_{16} \mathrm{O}_{3}[\mathrm{M}]^{+} 196.1099$, found: 196.1080 .<smiles>COC[C@H](O)[C@@H](CC(Cc1c(OC)cc(OC)cc1OC)OC)OC</smiles>

$(2 R, 3 R, 4 R, 5 S)-1,3,4,5-$ Tetramethoxy-6-(2,4,6-

trimethoxy-phenyl)-hexan-2-ol (2l). (Condition A (48 hours) provided 21 from 11 in $65 \%$ overall yield as colorless oil; Condition B (2.0 equiv. Sc(OTf $\left.)_{3}\right)$ provided $2 \mathbf{l}$ from $\mathbf{1 l}$ (8) Chaudhuri, N. C.; Kool, E. T. Tetrahedron Lett. 1995, 36, 1795. 
in $61 \%$ overall yield as colorless oil). $[\alpha]_{\mathrm{D}}^{20}+29.7\left(c 0.47, \mathrm{CHCl}_{3}\right) ;{ }^{1} \mathrm{H} \mathrm{NMR}\left(\mathrm{CDCl}_{3}\right.$, $400 \mathrm{MHz}): \delta 6.10(\mathrm{~s}, 2 \mathrm{H}), 3.79(\mathrm{~s}, 9 \mathrm{H}), 3.59(\mathrm{~m}, 1 \mathrm{H}) 3.54(\mathrm{~s}, 3 \mathrm{H}), 3.51(\mathrm{~m}, 1 \mathrm{H}), 3.41(\mathrm{~s}$, $3 \mathrm{H}), 3.38(\mathrm{~m}, 1 \mathrm{H}), 3.35(\mathrm{~s}, 3 \mathrm{H}), 3.27(\mathrm{~s}, 1 \mathrm{H}), 3.47(\mathrm{~m}, 2 \mathrm{H}), 3.14(\mathrm{~s}, 3 \mathrm{H}), 2.99$ (dd, $J=$ 13.2, 8.4 Hz, $1 \mathrm{H}), 2.86(\mathrm{dd}, J=8.4,4.8 \mathrm{~Hz}, 1 \mathrm{H}) ;{ }^{13} \mathrm{C} \mathrm{NMR}\left(75 \mathrm{MHz}, \mathrm{CDCl}_{3}\right): \delta 159.7$, 159.0, 107.1, 90.4, 80.6, 78.7, 77.4, 73.8, 71.1, 59.3, 59.1, 58.1, 57.4, 55.5, 55.2, 23.4; IR (film) $v_{\max }$ 3483, 2930, 2832, 1610, $1460 \mathrm{~cm}^{-1}$; HRMS(CI, Na) $m / z$ calc'd for $\mathrm{C}_{19} \mathrm{H}_{32} \mathrm{NaO}_{8}[\mathrm{M}+\mathrm{Na}]^{+}$411.1995, found: 411.1845 .<smiles>COc1c(C[C@H](C)C[C@H](OC)[C@@H](O)CO)cc(Br)cc1OCc1ccccc1</smiles>

(2R,3S,5R)-6-(3-Benzyloxy-5-bromo-2-methoxyphenyl)-3-methoxy-5-methyl-hexane-1,2-diol (10b). (Condition A provided 10b from 9b in $63 \%$ overall yield as colorless oil); $[\alpha]_{\mathrm{D}}^{20}-1.8^{\circ}\left(c 0.80, \mathrm{CHCl}_{3}\right) ;{ }^{1} \mathrm{H} \mathrm{NMR}\left(\mathrm{CDCl}_{3}\right.$, $400 \mathrm{MHz}): \delta 7.50-7.30(\mathrm{~m}, 5 \mathrm{H}), 6.95(\mathrm{~d}, J=2.0 \mathrm{~Hz}, 1 \mathrm{H}), 6.88(\mathrm{~d}, J=2.4 \mathrm{~Hz}, 1 \mathrm{H}), 5.05$ (s, 2H), $3.80(\mathrm{~s}, 3 \mathrm{H}), 3.73(\mathrm{~m}, 2 \mathrm{H}), 3.65$ (t, $J=7.2 \mathrm{~Hz}, 1 \mathrm{H}), 3.44(\mathrm{~m}, 1 \mathrm{H}), 3.40(\mathrm{~s}, 3 \mathrm{H})$, $2.61(\mathrm{dd}, J=13.2,6.0 \mathrm{~Hz}, 1 \mathrm{H}), 2.37$ (dd, $J=13.2,8.4 \mathrm{~Hz}, 1 \mathrm{H}), 1.93$ (m, 1H), 1.64 (m, $1 \mathrm{H}), 1.22(\mathrm{~m}, 1 \mathrm{H}), 0.87(\mathrm{~d}, J=6.8 \mathrm{~Hz}, 3 \mathrm{H}) ;{ }^{13} \mathrm{C} \mathrm{NMR}\left(75 \mathrm{MHz}, \mathrm{CDCl}_{3}\right): \delta 152.5,147.0$, 136.5, 136.4, 128.6, 128.3, 127.4, 125.7, 115.7, 115.4, 81.4, 72.6, 70.9, 63.3, 60.6, 58.5, 37.8, 37.7, 30.7, 19.6; IR (film) $v_{\max } 3411,2931,1575,1471,1093 \mathrm{~cm}^{-1}$; HRMS(CI, Na) $m / z$ calc'd for $\mathrm{C}_{22} \mathrm{H}_{29} \mathrm{BrNaO}_{5}[\mathrm{M}+\mathrm{Na}]^{+} 475.1096$, found: 475.1096 . Found to be identical to previous reports of $\mathbf{1 0 b} .^{5}$<smiles>COc1cc([N+](=O)[O-])c(OC)c(C[C@@H](C)C[C@H](OC)[C@H](O)CO)c1OC</smiles>

(2R,3S,5R)-3-Methoxy-5-methyl-6-(2,3,6-trimethoxy5-nitrophenyl)hexane-1,2-diol (10a). (Condition A provided 10a from 9a in 84\% overall yield as light yellow oil); $[\alpha]_{\mathrm{D}}^{20}-14.8^{\circ}\left(c\right.$ 1.20, $\left.\mathrm{CHCl}_{3}\right) ;{ }^{1} \mathrm{H} \mathrm{NMR}(400 \mathrm{MHz}$, $\left.\mathrm{CDCl}_{3}\right) \delta 7.38(\mathrm{~s}, 1 \mathrm{H}), 3.90(\mathrm{~s}, 3 \mathrm{H}), 3.88(\mathrm{~s}, 3 \mathrm{H}), 3.82(\mathrm{~s}, 3 \mathrm{H}), 3.57(\mathrm{~m}, 2 \mathrm{H}), 3.5(\mathrm{~m}, 1 \mathrm{H})$, $3.40(\mathrm{~m}, 1 \mathrm{H}), 3.38(\mathrm{~s}, 3 \mathrm{H}), 2.65(\mathrm{dd}, J=13,6.5 \mathrm{~Hz}, 1 \mathrm{H}), 2.55(\mathrm{dd}, J=13,6.5 \mathrm{~Hz}, 1 \mathrm{H})$, 
$1.98(\mathrm{~m}, 1 \mathrm{H}), 1.59(\mathrm{~m}, 1 \mathrm{H}), 1.28(\mathrm{~m}, 1 \mathrm{H}), 0.86(\mathrm{~d}, J=6.8 \mathrm{~Hz}, 3 \mathrm{H}) ;{ }^{13} \mathrm{C} \mathrm{NMR}(75 \mathrm{MHz}$, $\left.\mathrm{CDCl}_{3}\right) \delta 152.8,148.7,147.8,138.5,130.8,107.3,81.5,73.0,63.4,62.5,60.9,58.4$ 56.2, 37.9, 32.5, 30.4, 19.7 ppm; IR (film) $v_{\max } 3450,2942,1485,1425 \mathrm{~cm}^{-1}$; HRMS(CI, Na) $m / z$ calc'd for $\mathrm{C}_{17} \mathrm{H}_{27} \mathrm{NNaO}_{8} \quad[\mathrm{M}+\mathrm{Na}]^{+} 396.1634$, Found 329.1638. Found to be identical to previous reports of $\mathbf{1 0 a}{ }^{9}$

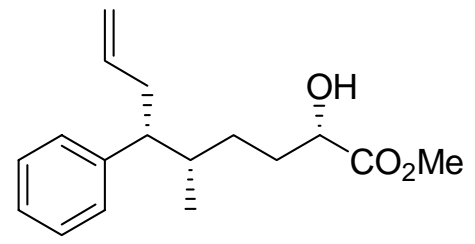

(2S,5S,6R)-methyl-2-hydroxy-5-methyl-6-phenylnon-8-

enoate (3a). (2S,5S,6S)-methyl-5-methyl-6-phenyl-tetrahydro-2H-pyran-2-carboxylate (1a) $(23.4 \mathrm{mg}, 0.10 \mathrm{mmol})$ was placed in a round-flask containing a stir bar, $2.0 \mathrm{~mL}$ $\mathrm{CH}_{2} \mathrm{Cl}_{2}$ and allyltrimethylsilane $(228.1 \mathrm{mg}, 2.0 \mathrm{mmol})$. To the solution $\mathrm{Sc}(\mathrm{OTf})_{3}(98.4$ $\mathrm{mg}, 0.20 \mathrm{mmol}$ ) was then added. The solution was stirred for 2 days before $5 \mathrm{~mL}$ water was added. The mixture was allowed to stir for 10 minutes then extracted with additional $\mathrm{CH}_{2} \mathrm{Cl}_{2}(3 \mathrm{ml} \times 3)$. The combined organic extracts were dried over $\mathrm{MgSO}_{4}$, filtered, and concentrated under reduced pressure. The residue was purified by flash chromatography over silica gel with (hexanes/EtOAc: $90 / 10)$ to yield $81 \%(\mathrm{dr}=1.7 / 1)$ of $(2 S, 5 S)$-methyl-2hydroxy-5-methyl-6-phenylnon-8-enoate $(22.4 \mathrm{mg}, 81.1 \mu \mathrm{mol})$ as light yellow oil. ${ }^{1} \mathrm{H}$ NMR ${ }^{1} \mathrm{H}$ NMR $\left(400 \mathrm{MHz}, \mathrm{CDCl}_{3}, 1.7: 1\right.$ diastereomeric ratio, asterisk denotes minor isomer peaks); $\delta 7.26(\mathrm{~m}, 5 \mathrm{H}), 7.17^{*}(\mathrm{~m}, 5 \mathrm{H}), 5.58(\mathrm{~m}, 1 \mathrm{H}), 5.56^{*}(\mathrm{~m}, 1 \mathrm{H}), 4.90(\mathrm{~m}$, $2 \mathrm{H}), 4.86^{*}(\mathrm{~m}, 2 \mathrm{H}), 4.16(\mathrm{~m}, 1 \mathrm{H}), 4.19^{*}(\mathrm{~m}, 1 \mathrm{H}), 3.67(\mathrm{~s}, 3 \mathrm{H}), 3.67 *(\mathrm{~s}, 3 \mathrm{H}), 2.56(\mathrm{~d}, J=$ $6.0 \mathrm{~Hz}, 1 \mathrm{H}), 2.62 *(\mathrm{~d}, J=8.4 \mathrm{~Hz}, 1 \mathrm{H}), 2.40(\mathrm{~m}, 2 \mathrm{H}), 2.38 *(\mathrm{~m}, 2 \mathrm{H}), 1.66(\mathrm{~m}, 3 \mathrm{H}), 1.60 *$ $(\mathrm{m}, 3 \mathrm{H}), 1.42(\mathrm{~m}, 2 \mathrm{H}), 1.44 *(\mathrm{~m}, 2 \mathrm{H}), 1.18(\mathrm{~m}, 1 \mathrm{H}), 1.20 *(\mathrm{~m}, 2 \mathrm{H}) 0.92(\mathrm{~d}, J=6.4 \mathrm{~Hz}$ $3 \mathrm{H}), 0.72 *(\mathrm{~d}, J=6.4 \mathrm{~Hz}, 3 \mathrm{H}) ;{ }^{13} \mathrm{C} \mathrm{NMR}\left(75 \mathrm{MHz}, \mathrm{CDCl}_{3}\right) \delta 175.6,137.3,128.6,127.9$, 125.9, 115.6, 70.5, 52.5, 51.3, 43.5, 37.5, 31.8, 29.5, 28.9, 16.9 ppm; IR (film) $v_{\max } 3487$, 2955, 2239, 1736, 1446, $1221 \mathrm{~cm}^{-1}$; $\operatorname{HRMS}(\mathrm{CI}, \mathrm{Na}) \mathrm{m} / z$ calc'd for $\mathrm{C}_{17} \mathrm{H}_{24} \mathrm{NaO}_{3}[\mathrm{M}+\mathrm{Na}]^{+}$ 299.1623, Found 299.1627.

(9) Andrus M. B.; Meredith E. L.; Sekhar B. B. V. S. Org. Lett. 2001, 3, 259. 
<smiles>CC(=O)[C@H](O)CC[C@H](C)[C@H](C#N)c1ccccc1</smiles>

\section{(2S,5S,6R)-methyl-6-azido-2-hydroxy-5-methyl-6-}

phenylhexanoate (3b). (2S,5S,6S)-methyl-5-methyl-6-phenyl-tetrahydro-2H-pyran-2carboxylate (1a) $(23.4 \mathrm{mg}, 0.10 \mathrm{mmol})$ was placed in a microwave tube containing a stir bar, $1.0 \mathrm{~mL} \mathrm{CH}_{2} \mathrm{Cl}_{2}$ and $\mathrm{TMSN}_{3}(230.1 \mathrm{mg}, 2.01 \mathrm{mmol})$. To the solution $\mathrm{Sc}(\mathrm{OTf})_{3}(98.4$ $\mathrm{mg}, 0.20 \mathrm{mmol}$ ) was then added. The tube was sealed and irradiated in a microwave (300 watts, 70 PSI and $100{ }^{\circ} \mathrm{C}$ ) for 60 minutes before water was added. The mixture was allowed to stir for 10 minutes then extracted with additional $\mathrm{CH}_{2} \mathrm{Cl}_{2}(3 \mathrm{ml} \times 3)$. The combined organic extracts were dried over $\mathrm{MgSO}_{4}$, filtered, and concentrated under reduced pressure. The residue was purified by flash chromatography over silica gel with (hexanes/EtOAc: $85 / 15)$ to yield $91 \%(\mathrm{dr}=1.4 / 1)$ of $(2 S, 5 \mathrm{~S})$-methyl-6-azido-2-hydroxy-5methyl-6-phenylhexanoate $(25.3 \mathrm{mg}, 91.1 \mu \mathrm{mol})$ as light yellow oil. ${ }^{1} \mathrm{H}$ NMR $(400 \mathrm{MHz}$, $\mathrm{CDCl}_{3}, 1.4: 1$ diastereomeric ratio, asterisk denotes minor isomer peaks); $\delta 7.36(\mathrm{~m}$, $5 \mathrm{H}), 7.21 *(\mathrm{~m}, 5 \mathrm{H}), 4.20(\mathrm{~m}, 1 \mathrm{H}), 4.22 *(\mathrm{~m}, 1 \mathrm{H}), 3.72(\mathrm{~s}, 3 \mathrm{H}), 3.76 *(\mathrm{~s}, 3 \mathrm{H}), 2.78(\mathrm{~d}, J=$ $5.2 \mathrm{~Hz}, 1 \mathrm{H}), 2.69 *(\mathrm{~d}, J=8.6 \mathrm{~Hz}, 1 \mathrm{H}), 1.86(\mathrm{~m}, 2 \mathrm{H}), 1.81^{*}(\mathrm{~m}, 2 \mathrm{H}), 1.75(\mathrm{~m}, 2 \mathrm{H}), 1.69^{*}$ $(\mathrm{m}, 2 \mathrm{H}), 1.40(\mathrm{~m}, 1 \mathrm{H}), 1.40^{*}(\mathrm{~m}, 1 \mathrm{H}), 1.19(\mathrm{~m}, 1 \mathrm{H}), 1.08^{*}(\mathrm{~m}, 1 \mathrm{H}), 0.92(\mathrm{~d}, J=6.4 \mathrm{~Hz}$, $3 \mathrm{H}) ; 0.72 *(\mathrm{~d}, J=6.4 \mathrm{~Hz}, 3 \mathrm{H}) ;{ }^{13} \mathrm{C} \mathrm{NMR}\left(75 \mathrm{MHz}, \mathrm{CDCl}_{3}\right) \delta 175.4,138.6,128.8,128.1$, 127.7, 71.7, 70.2, 52.5, 38.3, 31.6, 28.5, $15.3 \mathrm{ppm}$; IR (film) $v_{\max } 3480,2955,2098$, 1736, 1453, $1249 \mathrm{~cm}^{-1}$; HRMS(CI, Na) $\mathrm{m} / z$ calc'd for $\mathrm{C}_{14} \mathrm{H}_{19} \mathrm{~N}_{3} \mathrm{NaO}_{3}[\mathrm{M}+\mathrm{Na}]^{+}$ 300.1324, Found 300.1328.

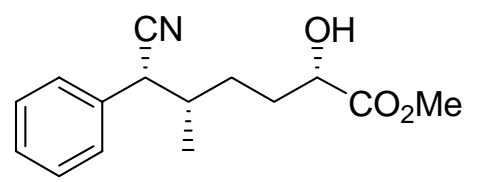

(2S,5S,6R)-methyl-6-cyano-2-hydroxy-5-methyl-6-

phenylhexanoate (3c). $\quad(2 S, 5 S, 6 S)$-methyl-5-methyl-6-phenyl-tetrahydro-2H-pyran-2carboxylate (1a) $(23.4 \mathrm{mg}, 0.10 \mathrm{mmol})$ was placed in a microwave tube containing a stir bar, $1.0 \mathrm{~mL} \mathrm{CH} \mathrm{Cl}_{2}$ and TMSCN (49.5 mg, $0.50 \mathrm{mmol}$ ). To the solution $\mathrm{Sc}(\mathrm{OTf})_{3}(98.4$ $\mathrm{mg}, 0.20 \mathrm{mmol}$ ) was then added. The tube was sealed and irradiated in a microwave (300 watts, 70 PSI and $100{ }^{\circ} \mathrm{C}$ ) for 20 minutes before water was added. The mixture was 
Mild Reductive Opening of Aryl Pyranosides Promoted by Scandium (III) Triflate

allowed to stir for 10 minutes then extracted with additional $\mathrm{CH}_{2} \mathrm{Cl}_{2}(3 \mathrm{ml} \times 3)$. The combined organic extracts were dried over $\mathrm{MgSO}_{4}$, filtered, and concentrated under reduced pressure. The residue was purified by flash chromatography over silica gel with (hexanes/EtOAc: $85 / 15)$ to yield $43 \%(\mathrm{dr}=4 / 1)$ of $(2 S, 5 \mathrm{~S})$-methyl-6-cyano-2-hydroxy-5methyl-6-phenylhexanoate $(11.3 \mathrm{mg}, 43.1 \mu \mathrm{mol})$ as light yellow oil. ${ }^{1} \mathrm{H}$ NMR ${ }^{1} \mathrm{H}$ NMR (400 $\mathrm{MHz}, \mathrm{CDCl}_{3}, 4: 1$ diastereomeric ratio, asterisk denotes minor isomer peaks); $\delta 7.38$ $(\mathrm{m}, 5 \mathrm{H}), 7.24^{*}(\mathrm{~m}, 5 \mathrm{H}), 4.17(\mathrm{~m}, 1 \mathrm{H}), 4.14^{*}(\mathrm{~m}, 1 \mathrm{H}), 3.82(\mathrm{~d}, J=6.4 \mathrm{~Hz}, 1 \mathrm{H}), 3.66^{*}(\mathrm{~d}$, $J=10.4 \mathrm{~Hz}, 1 \mathrm{H}), 3.77(\mathrm{~s}, 3 \mathrm{H}), 3.72 *(\mathrm{~s}, 3 \mathrm{H}), 2.76(\mathrm{~d}, J=4.8 \mathrm{~Hz}, 1 \mathrm{H}), 2.70 *(\mathrm{~d}, J=9.6$ $\mathrm{Hz}, 1 \mathrm{H}), 1.96(\mathrm{~m}, 1 \mathrm{H}), 2.00 *(\mathrm{~m}, 1 \mathrm{H}), 1.81(\mathrm{~m}, 1 \mathrm{H}), 1.79 *(\mathrm{~m}, 1 \mathrm{H}), 1.76(\mathrm{~m}, 2 \mathrm{H}), 1.75^{*}$ $(\mathrm{m}, 2 \mathrm{H}), 1.49(\mathrm{~m}, 1 \mathrm{H}), 1.42 *(\mathrm{~m}, 1 \mathrm{H}), 0.97(\mathrm{~d}, J=6.4 \mathrm{~Hz}, 3 \mathrm{H}), 1.02 *(\mathrm{~d}, J=6.4 \mathrm{~Hz}, 3 \mathrm{H})$; ${ }^{13} \mathrm{C}$ NMR $\left(75 \mathrm{MHz}, \mathrm{CDCl}_{3}\right) \delta 184.6,175.3,134.7,128.8,127.7,119.2,70.1,52.7,43.5$, 38.3, 31.7, 30.2, $15.6 \mathrm{ppm}$; IR (film) $v_{\max } 3476,2925,2239,1736,1494,1451 \mathrm{~cm}^{-1}$; $\operatorname{HRMS}(\mathrm{CI}, \mathrm{Na}) m / z$ calc'd for $\mathrm{C}_{15} \mathrm{H}_{19} \mathrm{NNaO}_{3}[\mathrm{M}+\mathrm{Na}]^{+}$284.1263, Found 284.1261.

\section{Assignment of Stereochemistry of Azide 3b}

The stereochemistry of the benzylic position in the resulting acyclic products found in table 3 was determined through a two step sequence; reduction and lactam formation of $\mathbf{3 b}$. Upon chromatographic separation, each diastereomer was fully characterized to secure the correct stereochemical assignment of the opened products (See below for details). Analysis of the threebond coupling constants $\left({ }^{3} \mathrm{~J}_{\mathrm{H} 1, \mathrm{H} 2}=4.2 \mathrm{~Hz}\right)$ correlating to the $\mathrm{C} 5$ and $\mathrm{C} 6$ stereogenic centers in the ${ }^{1} \mathrm{H}-\mathrm{NMR}$ spectrum of the "major" lactam permitted the stereochemical assignment as a cis configuration. Analysis of the three-bond coupling constants $\left({ }^{3} \mathrm{~J}_{\mathrm{H} 1, \mathrm{H} 2}=11.2 \mathrm{~Hz}\right)$ correlating to the C5 and C6 stereogenic centers in the ${ }^{1} \mathrm{H}-\mathrm{NMR}$ spectrum of the "minor" lactam permitted the stereochemical assignment as a trans configuration. In addition, a NOESY 1D was performed on the $\mathrm{C} 6$ protons of both isomers giving responses supporting the three-bond coupling constants described above (See below for observed n.O.e responses). The absolute stereochemistry of the 
C2 hydroxyl group and the C5 methyl group is based on the mechanism previously described for the [4+2]-annulation. ${ }^{1}$ The remaining two examples in table 3 (3a and $\mathbf{3 c}$ ) were assigned by analogy with $\mathbf{3 b}$.

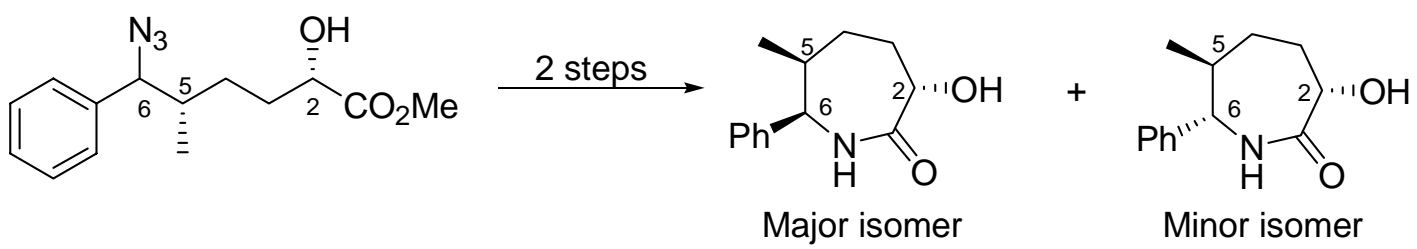

Azide 3b (110.0 mg, $0.397 \mathrm{mmol})$ was dissolved in THF (2.0 mL) followed by the addition of $10 \%$ palladium on carbon $(25.0 \mathrm{mg})$. The reaction was stirred under an atmosphere over 12 hours. The mixture was filtered through silica gel and concentrated to obtain a yellow colored oil ca. $93.0 \mathrm{mg}(93 \%)$. The amine was dissolved in benzene (5.0 $\mathrm{mL}$ ) and heated to $90^{\circ} \mathrm{C}$ for 7 days. The reaction was concentrated and the residue was purified by flash chromatography to over silica gel $\left(\mathrm{CHCl}_{3} / \mathrm{MeOH}\right.$ : $99 / 1$ to $\left.95 / 5\right)$ to yield the desired lactams $42.0 \mathrm{mg}$ (46\%) "major isomer" and $35.0 \mathrm{mg}(43 \%)$ "minor isomer".

“Major isomer" $(3 S, 6 S, 7 R)-3-H y d r o x y-6-m e t h y l-7-p h e n y l a z e p a n-2-o n e ; ~[\alpha]_{D}^{20}-2.4(c$ 1.3, $\mathrm{CHCl}_{3}$ ); colorless oil; ${ }^{1} \mathrm{H} \mathrm{NMR}\left(400 \mathrm{MHz} \mathrm{CDCl}_{3}\right) \delta 7.30(\mathrm{~m}, 5 \mathrm{H}), 6.57(\mathrm{~s}, 1 \mathrm{H})$, $4.79(\mathrm{~d}, J=4.2 \mathrm{~Hz}, 1 \mathrm{H}), 4.29(\mathrm{~d}, J=9.6 \mathrm{~Hz}, 1 \mathrm{H}), 3.92(\mathrm{~s}, 1 \mathrm{H}), 2.16(\mathrm{~m}, 1 \mathrm{H}), 1.93$ (m, 2H), $1.69(\mathrm{~m}, 2 \mathrm{H}), 1.08(\mathrm{~d}, J=7.6 \mathrm{~Hz}, 3 \mathrm{H}) ;{ }^{13} \mathrm{C} \mathrm{NMR}\left(75 \mathrm{MHz}, \mathrm{CDCl}_{3}\right) \delta 177.7,139.0$, 128.6, 127.5, 127.1, 71.6, 60.0, 38.9, 29.8, 28.7, 17.9 ppm; IR (film) $v_{\max } 3415,3216$, 2925, 1653, 1457, $1332 \mathrm{~cm}^{-1}$; HRMS(CI, NH3) $\mathrm{m} / z$ calc'd for $\mathrm{C}_{14} \mathrm{H}_{19} \mathrm{O}_{3}[\mathrm{M}+\mathrm{Na}]^{+}$ 242.1157, Found 242.1157.

“Minor isomer" (3S,6S,7S)-3-Hydroxy-6-methyl-7-phenylazepan-2-one; $[\alpha]_{D}^{20}-15.7$ (c 1.6, $\mathrm{CHCl}_{3}$ ); white solid, mp 122-123 ${ }^{\circ} \mathrm{C} ;{ }^{1} \mathrm{H} \mathrm{NMR}\left(400 \mathrm{MHz}, \mathrm{CDCl}_{3}\right) \delta 7.32(\mathrm{~m}, 3 \mathrm{H})$, $7.22(\mathrm{~m}, 2 \mathrm{H}), 5.85(\mathrm{~s}, 1 \mathrm{H}), 4.28(\mathrm{~d}, J=11.2 \mathrm{~Hz}, 1 \mathrm{H}), 4.06(\mathrm{~s}, 1 \mathrm{H}), 3.98(\mathrm{dd}, J=9.6,4.0$ 
$\mathrm{Hz}, 1 \mathrm{H}), 2.12(\mathrm{~m}, 2 \mathrm{H}), 1.98(\mathrm{~m}, 1 \mathrm{H}), 1.74(\mathrm{~m}, 1 \mathrm{H}), 1.64(\mathrm{~m}, 1 \mathrm{H}), 0.53(\mathrm{~d}, J=6.4 \mathrm{~Hz}$, $3 \mathrm{H}) ;{ }^{13} \mathrm{C} \mathrm{NMR}\left(75 \mathrm{MHz}, \mathrm{CDCl}_{3}\right) \delta 177.0,140.8,129.2,128.4,126.8,70.1,63.9,38.9$, 36.5, 33.1, 20.3 ppm; IR (film) $v_{\max } 3413,3216,2925,1653,1456,1330 \mathrm{~cm}^{-1}$; HRMS(CI, NH3) $m / z$ calc'd for $\mathrm{C}_{14} \mathrm{H}_{19} \mathrm{O}_{3}[\mathrm{M}+\mathrm{Na}]^{+} 242.1157$, Found 242.1157.

\section{Observed n.O.e's for both "Major" and "Minor" lactams.}

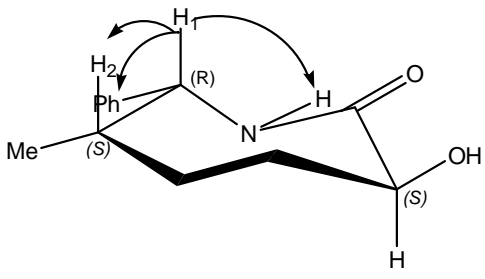

"Major isomer"

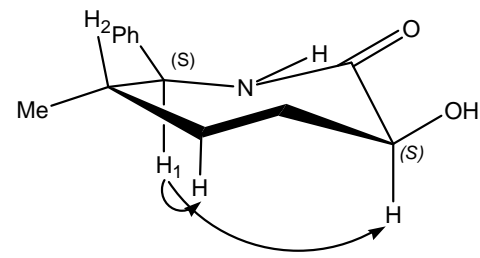

"Minor isomer" 